\title{
Ribs of Pinna nobilis shell induce unexpected microstructural changes that provide unique mechanical properties
}

\author{
Kinga Nalepka ${ }^{\mathrm{a}, *}$, Katarzyna Berent ${ }^{\mathrm{b}}$, Antonio G. Checa ${ }^{\mathrm{c}, \mathrm{d}}$, Tomasz Machniewicz ${ }^{\mathrm{a}}$,

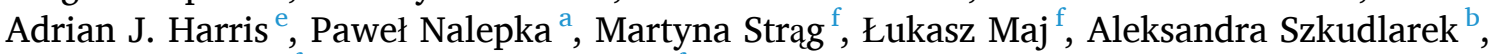 \\ Magdalena Bieda ${ }^{\mathrm{f}}$, Krzysztof Sztwiertnia ${ }^{\mathrm{f}}$ \\ ${ }^{\text {a }}$ Faculty of Mechanical Engineering and Robotics, AGH University of Science and Technology, Krakow, Poland \\ ${ }^{\mathrm{b}}$ Academic Centre for Materials and Nanotechnology, AGH University of Science and Technology, Krakow, Poland \\ ${ }^{\mathrm{c}}$ Department of Stratigraphy and Paleontology, University of Granada, Granada, Spain \\ ${ }^{\mathrm{d}}$ Andalusian Institute of Earth Sciences, CSIC-University of Granada, Armilla, Spain \\ e Micro Materials Ltd., Willow House, Ellice Way, Yale Business Village, Wrexham, LL13 7YL, UK \\ ${ }_{\mathrm{f}}^{\mathrm{f}}$ Institute of Metallurgy and Materials Science, Polish Academy of Sciences, Krakow, Poland
}

\section{A R T I C L E I N F O}

\section{Keywords:}

Biogenic calcite

EBSD

Twinning

Mechanical anisotropy

Nano-indentation

Compressive strength

\begin{abstract}
A B S T R A C T
The reinforcement function of shell ribs depends not only on their vaulted morphology but also on their microstructure. They are part of the outer layer which, in the case of the Pinna nobilis bivalve, is built from almost monocrystalline calcitic prisms, always oriented perpendicular to the growth surfaces. Originally, prisms and their $c$-axes follow the radii of rib curvature, becoming oblique to the shell thickness direction. Later, prisms bend to reach the nacre layer perpendicularly, but their $c$-axes retain the initial orientation. Calcite grains form nonrandom boundaries. Most often, three twin disorientations arise, with two of them observed for the first time. Nano-indentation and impact tests demonstrate that the oblique orientation of $c$-axes significantly improves the hardness and fracture toughness of prisms. Moreover, compression tests reveal that the rib area achieves a unique strength of $700 \mathrm{MPa}$. The detection of the specific microstructure formed to toughen the shell is novel.
\end{abstract}

\section{Introduction}

Bivalve shells are hierarchically complex biocomposites consisting of calcium carbonate (calcite or aragonite) and an organic matrix [1-3]. They have been improved over millions of years of evolution to provide effective protection against predators. Consequently, the shells are lightweight and exhibit outstanding mechanical properties compared to the materials from which they are built. Thus, protective armors are an excellent source of inspiration for the formation of biomimetic engineering materials with an equally unique combination of high strength and fracture toughness [4-7]. Reproducing the shell's microstructure requires in-depth knowledge of it as well as of the mechanical properties of the components $[8,9]$. The present work is part of the search for engineering materials inspired by nature. The subject is a shell of the Pinna nobilis species with an unusual morphology due to the repair of an extensive injury that occurred at a submature stage. Thus, the examined specimen is distinguished by a large, strongly ribbed surface.
The Mediterranean pen shell Pinna nobilis Linnaeus, 1758 (superfamily Pinnoidea, Order Ostreida [10]) is a large endobyssate bivalve species that lives with its anterior end buried within the sediment. The shell is ornamented with radial ribs that periodically produce scales directed towards the margin (see App. A). The shell wall is made of two layers. The outer one has a columnar calcite prismatic (CCP) microstructure: large polygonal prismatic grains elongated perpendicular to the outer shell surface, surrounded by organic sheaths. The inner layer is made of nacre, which extends for less than half the anteroposterior diameter (e.g. Refs. $[1,3,11]$ ). The two layers perform different functions. The thick $(1-5 \mu \mathrm{m})$ periprismatic organic membranes make the outer prismatic layer particularly flexible [12]. When the two valves abut, tight sealing [13-15] is achieved by flexible deformation of the wide prismatic margins. With progressive thickening of the prismatic layer towards the apical end (up to several $\mathrm{mm}$ in large specimens), flexing becomes hindered and the prismatic layer exerts an armoring function. The radial ribs developed on the prismatic layer contribute to

\footnotetext{
* Corresponding author.

E-mail address: knalepka@agh.edu.pl (K. Nalepka).
} 
reinforcement. Given the ability of the animal to retract deeply inside the shell, damage to the margin is not usually lethal. Additional protection is provided by the tough and rigid nacre layer.

Each prismatic unit is characterized by low disorientation (the term is explained in Sec. 6) and therefore becomes a grain similar to a monocrystal (e.g. Refs. [13,14,16-18]). Previous studies on flat shell areas report that the $c$-axes of prismatic grains remain parallel to their long axes [19-22]. The latter characteristic is defined as a physical axis built from the gravity centers of the successive cross sections of the prismatic unit.

The CCP layers of bivalves have been widely studied [11,18,23-28]. However, their mechanical properties have received little attention compared to nacre [29-32] and crossed-lamellar microstructures [33, 34]. Strag et al. [35] reported values of $460 \mathrm{MPa}$ and $3.86 \mathrm{GPa}$ for the compressive strength and nanohardness of the calcitic layer of Pinctada margaritifera, respectively. Kunitake et al. [36] showed that the hardness of the Atrina rigida CCP layer varies with the rotation of the indenter tip around the normal to the loaded $c$ plane. Accordingly, the range of
3.47-4.19 GPa was obtained at a penetration depth of $170 \mathrm{~nm}$. A similar dependence was found in Pinna nobilis: $3.89-4.86 \mathrm{GPa}$ at a variable indentation depth of 100-250 nm [37].

The aim of this study is to accurately characterize the CCP microstructure of $P$. nobilis in the areas of ribs and link it with the mechanical properties of the constituent elements as well as with the response to load of a representative volume. A specimen with a large, densely ribbed surface was used, shaped by extensive damage in the early stage of growth. Investigations with application of the electron backscatter diffraction (EBSD) method have revealed unusual orientations of prismatic calcite grains, which play a key role in transferring the external load. This was demonstrated through theoretical analysis and then confirmed by the identification of mechanical properties at the local and global levels with nano-indentation, nano-impact and compression tests.

\section{Microstructure identification}

The studied shell has a morphology unusual for the $P$. nobilis species
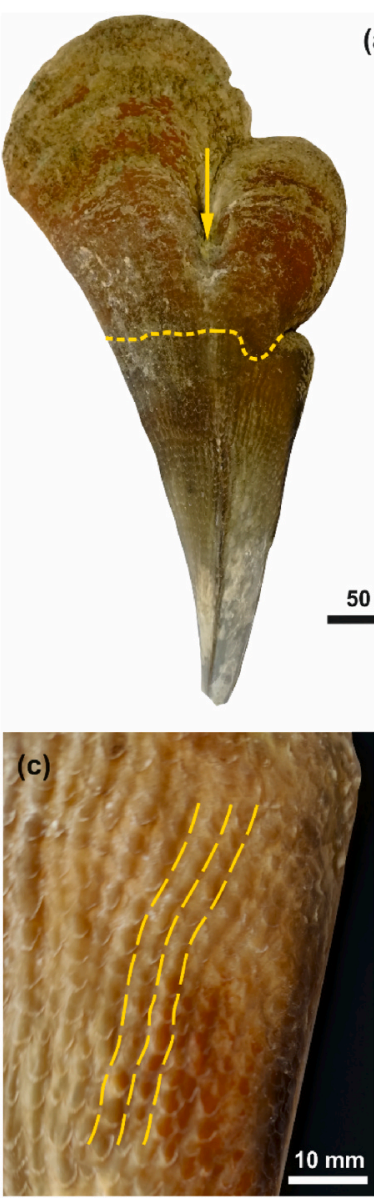

(a)
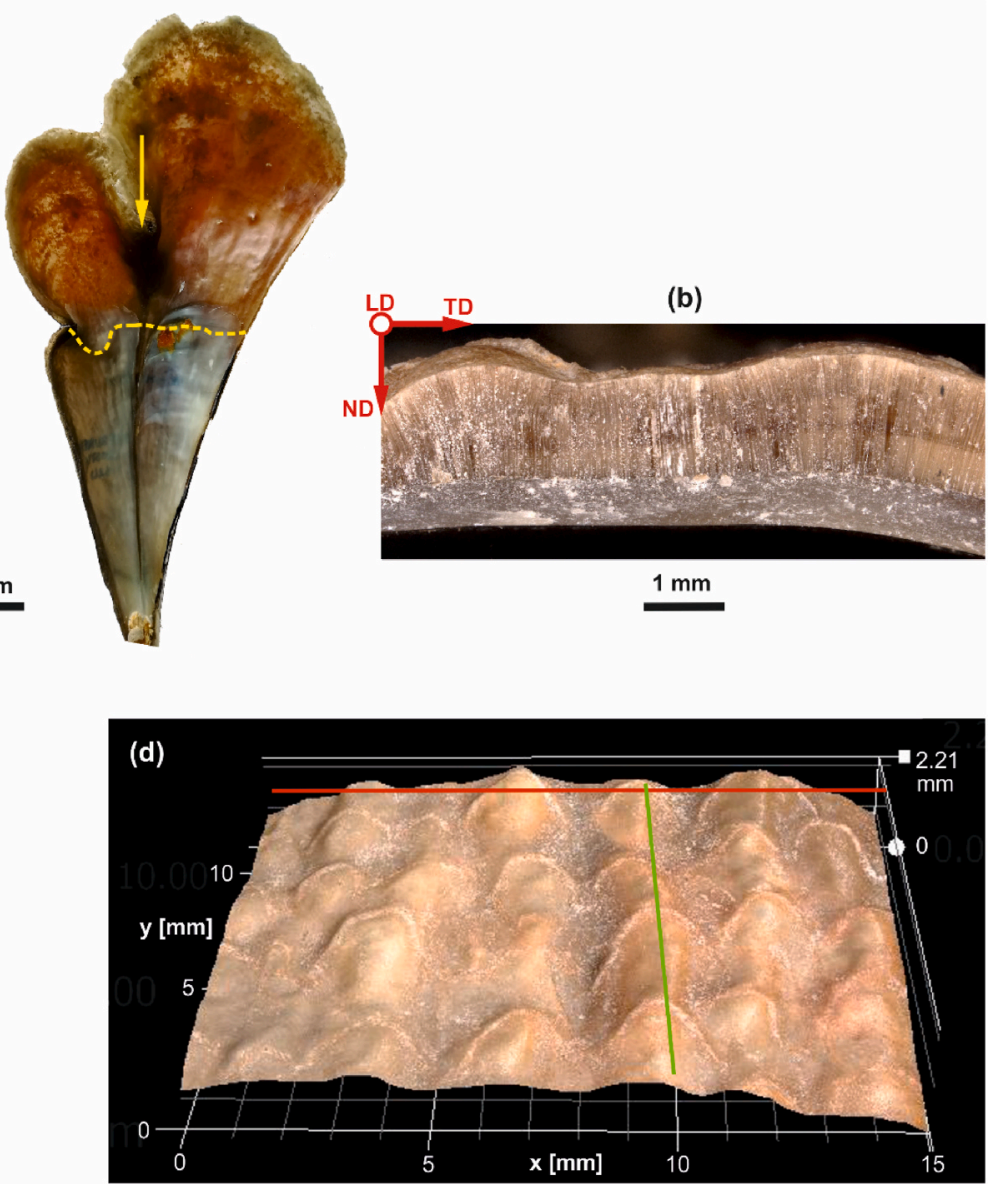

(e)

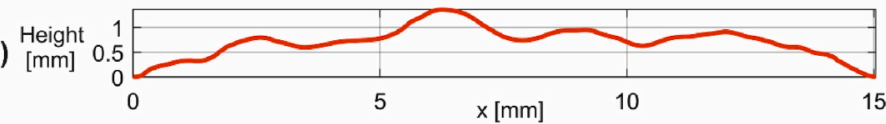

(f)

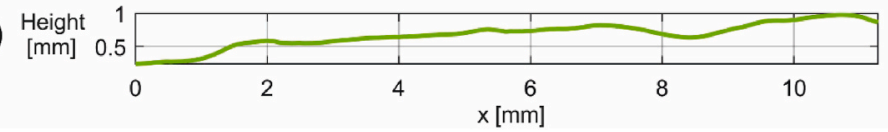

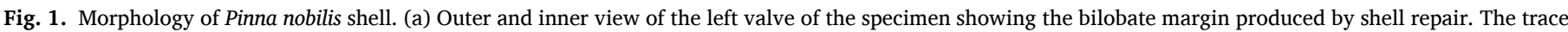

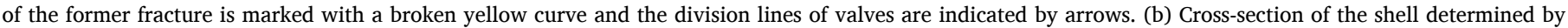

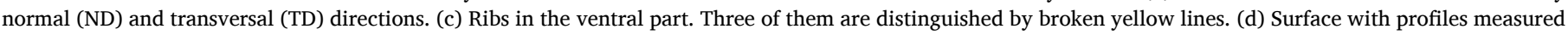

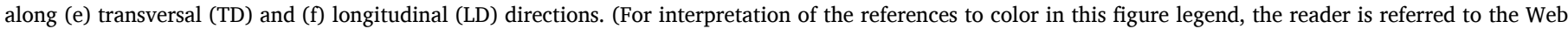
version of this article.) 
(compare App. A). A clear division line running longitudinally on both valves is visible (Fig. 1a). The reason is breakage of the shell at a submature stage of growth. The trace of the former fracture begins with a clear notch separating the posterior of the primary shell from the restored area. The further course of the crack is determined, on the external surface, by the discontinuity of the ribs, which are distributed more densely in the repaired part. In the same area, there is a thick nacre coating on the inside. After breaking, the shell was rebuilt asymmetrically. The dorsal and ventral parts developed independently. The first of them underwent significant expansion. In spite of the bilobate appearance, the examined shell preserves the geometric structure of the surface characteristic for the $P$. nobilis species. Its key component is the waviness generated by ribs running in the radial direction (Fig. 1b-e). They are formed by periodically distributed segments of mantle specialized for
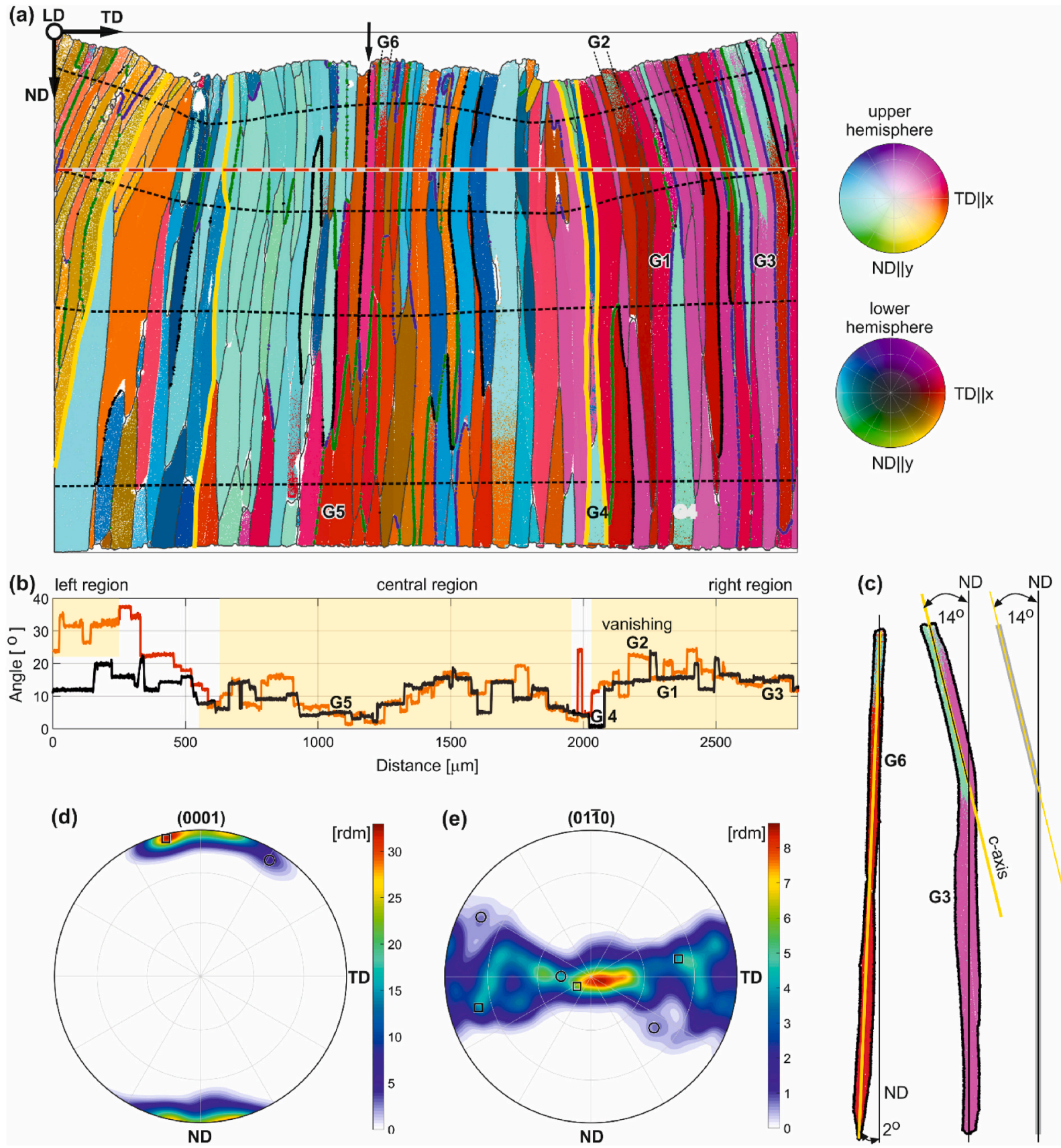

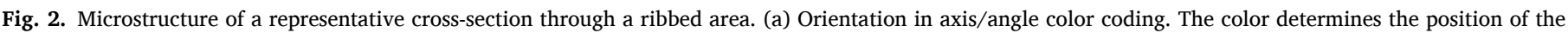

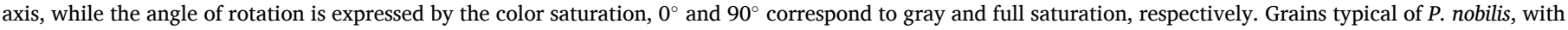

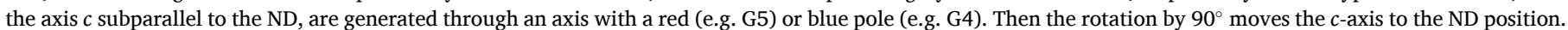

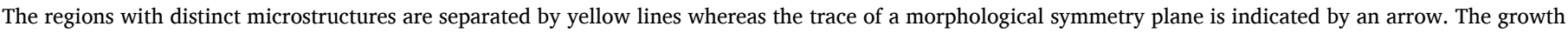

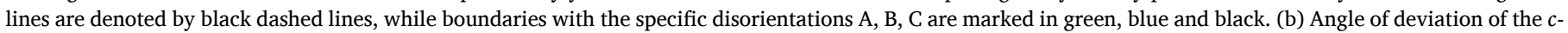

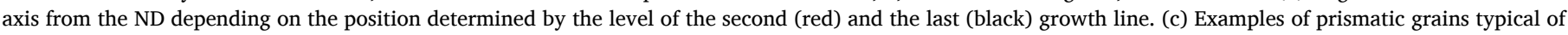

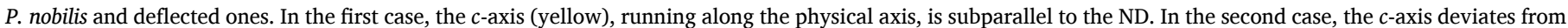

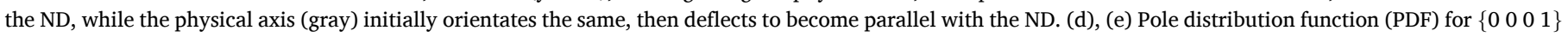

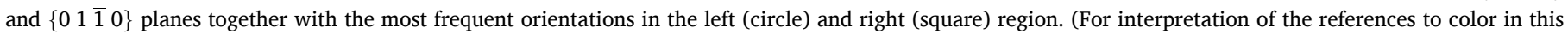
figure legend, the reader is referred to the Web version of this article.) 
the production of ribs. Upon growth, segments move along radial trajectories, thus producing a series of radial ribs [38]. The waviness integrally inscribed in the three-dimensional surface topography induces a specific microstructure of the shell's cross-section. In order to identify it, in-depth EBSD investigations were carried out.

Prismatic grains, growing perpendicularly to the undulated outer surface, deviate from the normal direction (ND) determined by the shell thickness. As a result, in the outer layer, the directions of fast growth, i.e. $c$-axes of calcite grains, are mostly oblique (Fig. 2). With shell thickening, growth lines become flatter and the orientation of the physical axes becomes more and more parallel to ND. This process leads to the deflection of prismatic grains as well as to elimination of those that exhibit a significant initial deviation from the normal direction (Fig. 2a).

The ordered orientation of physical axes is not transferred to calcite $c$-axes. It turns out that, during the growth of grains, the $c$-axes maintain their initial direction. As a result, obliquely initiated orientation becomes obligatory throughout the entire thickness of the shell. Thus, the wavy surface determines the texture of the entire protective armor. The $c$-axis, instead of taking the direction normal to the nacre layer (ND), creates a coherent structure by deviating by several or even several dozen degrees from the ND. Analysis of the EBSD results reveals a clear division into three areas. Both the outer surface profile and the growth lines indicate that these regions correspond to three adjacent ribs running perpendicular to the image in the longitudinal direction (LD) (see Fig. 2a). The middle area is initiated by a local shell elevation with a small curvature, while the lateral ones begin with a greater slope. As a result, in the central region, the $c$-axes of grains are most frequently tilted by a small angle of $12^{\circ}$ relative to the ND, and their initial orientation is maintained up to the nacre layer (Fig. 2b, compare red and black plots). The central area is flanked by two half ribs with a higher curvature, particularly the left one. Hence, in their upper parts, the $c$ axes of grains most often orientate at an angle of $32^{\circ}$ (left rib) and $14^{\circ}$ (right rib) relative to the ND (Fig. 2b, red plot). The organization of both zones is similar. Initially, grains with an oblique inclination tend to disappear, whereas their neighbors expand at their expense. In this way, grains with $c$-axes significantly deviated from the ND are eliminated, while those which form a group with a similar orientation of $c$-axes reach the nacre layer. The G1 and G2 grains are perfect examples. The first one exhibits the deviation angle of $15^{\circ}$, preferred in the right rib area. The other one has a much larger angle of $23^{\circ}$. Finally, G1 reaches the nacre layer while G2 vanishes. Even its sudden deflection causes only a slight change in the orientation of calcite - the difference remains within $2^{\circ}$.

The analysis carried out confirms that the waviness, i.e. a lowfrequency component of the surface topography, determines the texture of the CCP layer. This property is formed by fibers, which define parallelism between calcite $c$-axes and radii describing the curvature of large surface fragments. The key factor ordering the microstructure waviness remains recorded in the cross-section of the shell. Its profile is reproduced by the growth line closest to the outer surface (Fig. 2a). Contrary to the waviness, the high-frequency component of the topography, roughness, is of less importance. It characterizes the random geometry of scales with which the ribs are covered. The investigations using a contact profilometer showed that, in the transverse direction, the arithmetic average roughness $R_{a}$ amounts to $0.2262 \mu \mathrm{m}$, and in the longitudinal direction it is more than twice as high, with $R_{a}$ equal to $0.4819 \mu \mathrm{m}$. The difference is due to the distinct nature of the TD and LD surface profiles. The former is largely regular and the latter contains
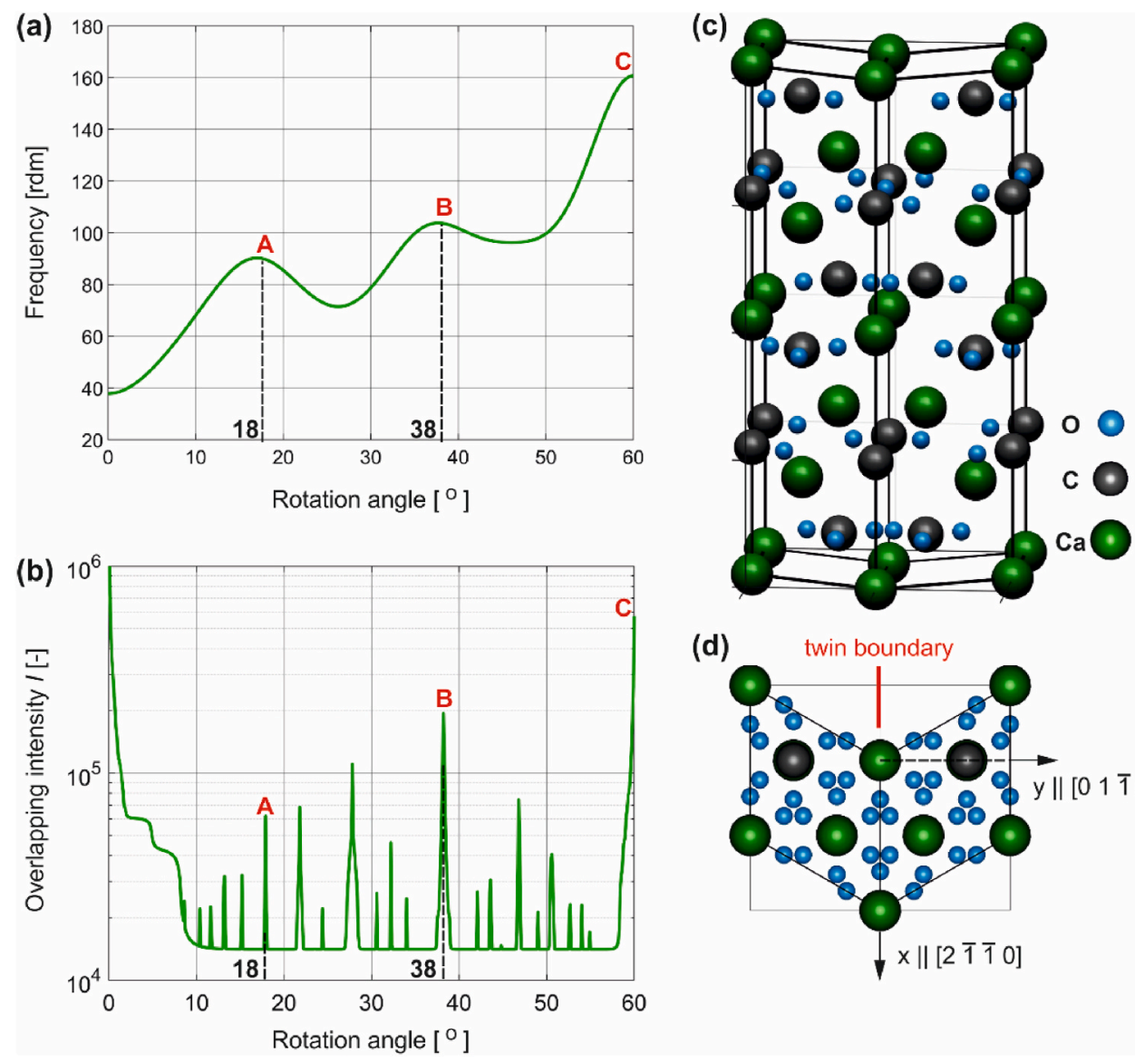

(d)

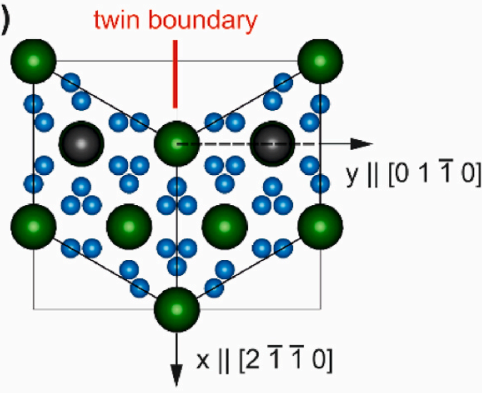

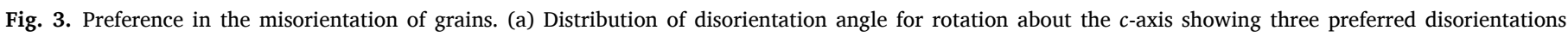

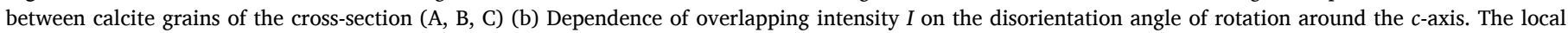
maxima determine low-energy disorientations. (c), (d) ( $01 \overline{1} 0)$ calcite twin. 
significant disturbances generated by faults of overlapping scales.

The question arises as to whether there is a preference in the mutual orientation of adjacent grains. The calculated Misorientation Distribution Function (MDF) shows that the grains rotate with respect to each other around the $c$-axis, taking three preferential positions. They are determined by the following values of the rotation angle $\omega: 18^{\circ}$ (A), $38^{\circ}$ (B) and $60^{\circ}$ (C) (Fig. 3a). High preference is usually due to the system's striving to the formation of low-energy boundaries. Hence, we conducted calculations to reveal how interfacial energy changes when adjacent calcite crystallites are rotated around the $c$-axis. Accurate determination of the quantity for the continuous rotation is virtually impossible due to the excessively high computational cost. Therefore, an approximated method developed by Gautam and Howe [39] is used. According to it, interfacial energy decreases with the increase of the total intensity $I$ contained in the areas of overlapping diffraction reflections from neighboring crystallites. Thus, using the last quantity we can find disorientations, to which local energy minima correspond (see Sec. 6).
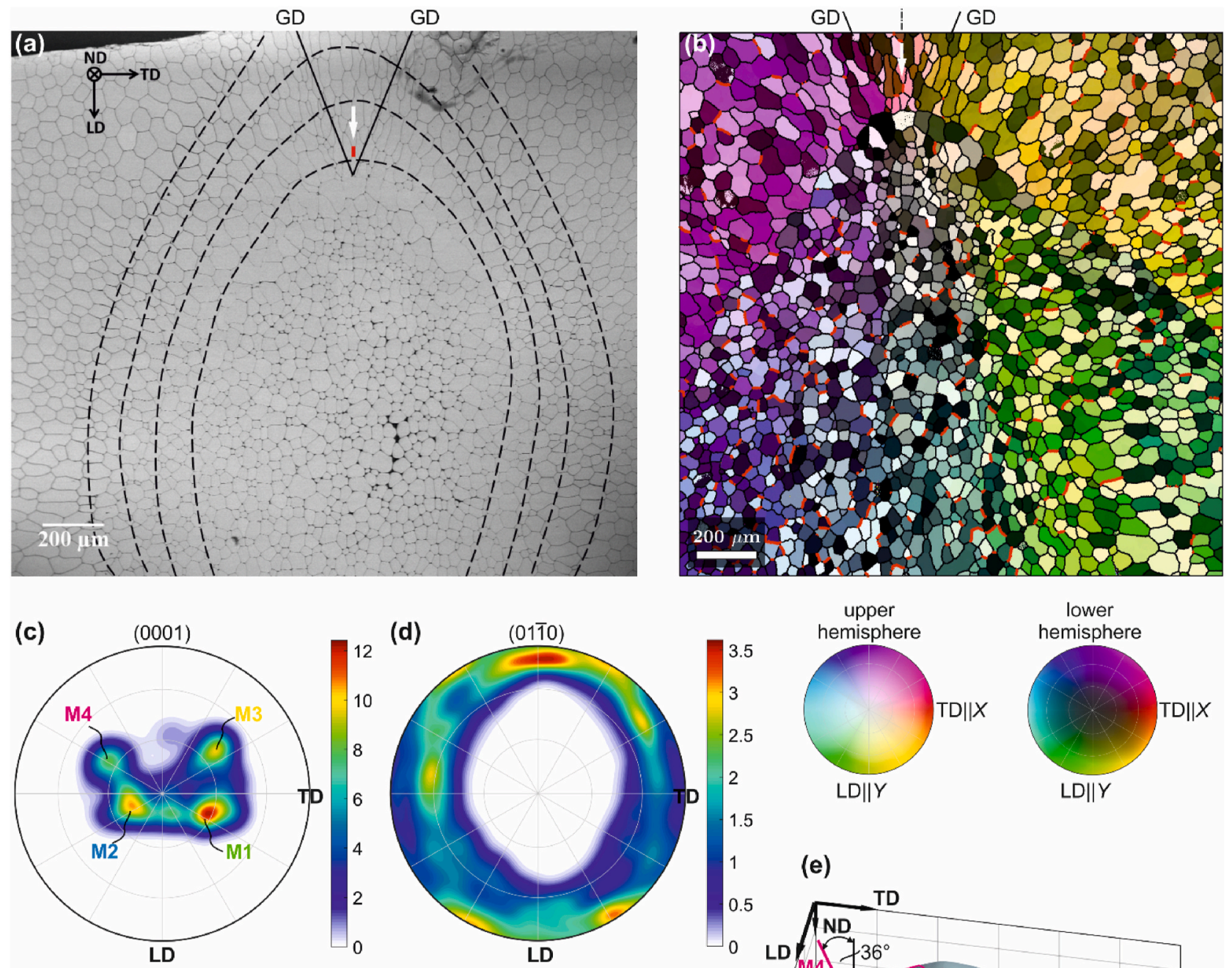

(f)
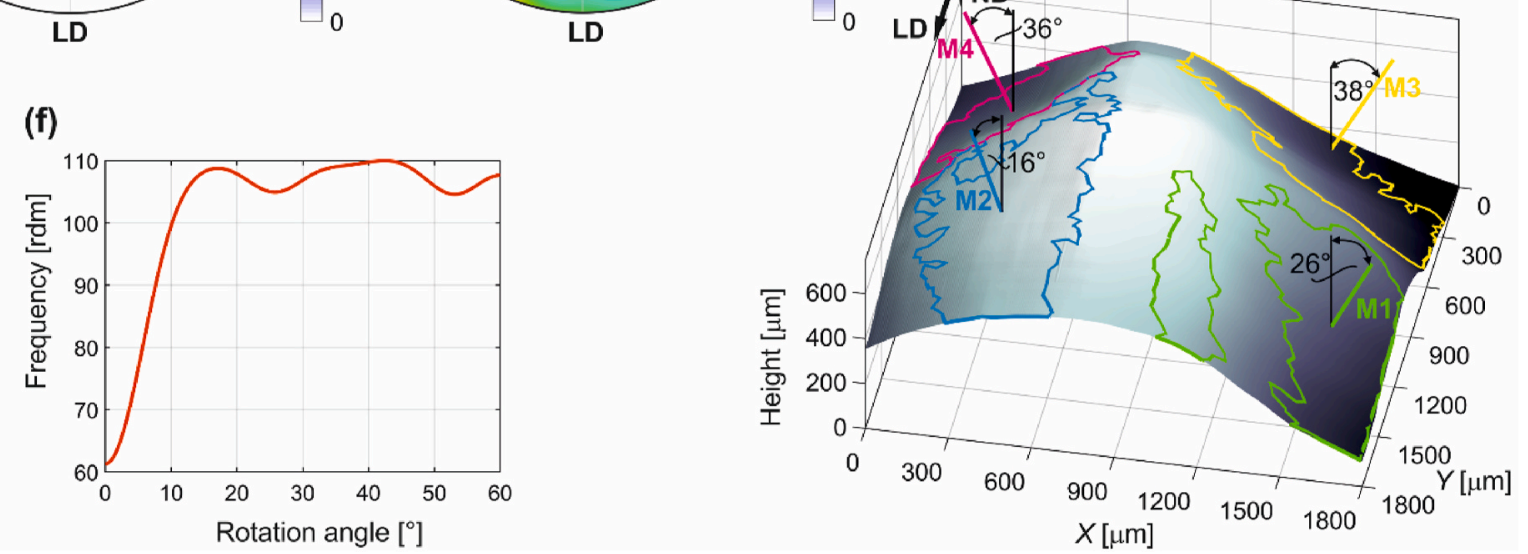

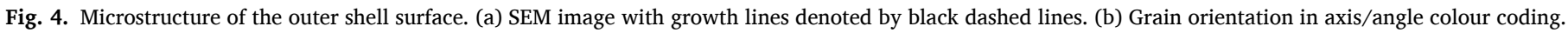

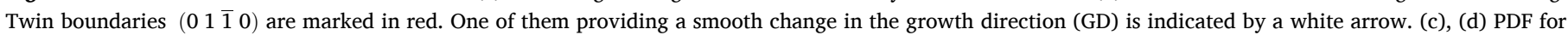

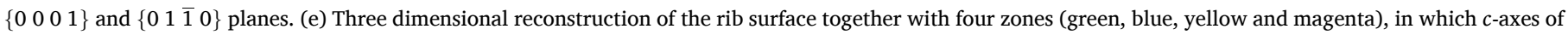

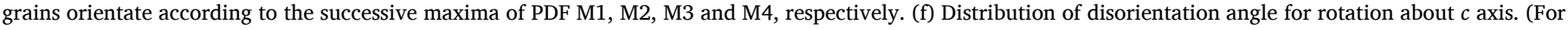
interpretation of the references to color in this figure legend, the reader is referred to the Web version of this article.) 
The results clearly show that strictly defined low-energy boundaries, i.e. rotation angles, are preferred (Fig. $3 \mathrm{~b}$ ). Particularly important is the disorientation described by the $60^{\circ}$ rotation about the $c$-axis. Two symmetrically equivalent orientation relationships correspond to it. They constitute two twin boundaries formed by the reflection planes

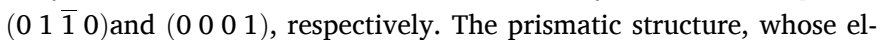
ements mostly run through the entire thickness of the shells, means that the first of them mainly occurs. The twin boundary ( $01 \overline{1} 0)$, positioning perpendicular to the growth line, allows it to be curved, because the crystallographic plane terminating the growth stage is mirrored to the adjacent area (comp. Fig. $3 c$ and $d$ and Fig. $4 a$ and b). As a result, grains separated by a morphological plane of symmetry form a coherent connection. Another example is the boundary running along the symmetry axis of the central region (indicated by the arrow in Fig. 2a). The other preferred disorientations are also generated by twin relationships, but this time the mirror planes have higher indices (1 $\overline{5} 40$ )and (47 $\overline{11} 0$ )for $38^{\circ}$ and $18^{\circ}$, respectively. These two uncovered twin relations are formed between grains of biogenic calcite thanks to the presence of biomolecules. They define new, hitherto unknown, orientation relationships that are highly prevalent in $P$. nobilis shells. The preferences observed experimentally are justified by the theoretical analysis of the misorientation space of a calcite crystal.

Something that could be of interest is how twin relationships can exist when calcite grains are separated by thick organic membranes. Checa et al. [27] showed how new membranes are introduced very early during the growth of grains in the CCP layers of several pterioids, thus dividing otherwise continuous crystalline domains. We can hypothesize that the low-energy disorientations were formed in initial growth stages in Pinna and twinned grains were later separated by membranes. Given the low disorientation recorded in Pinna grains, the twin relationship is preserved long after the neighboring grains are separated by the membrane.

The EBSD investigations performed for the representative fragment of the shell surface confirm that the waviness leads to the inclination of prismatic grains (Fig. 4). The $c$-axes oriented perpendicular to the curved surface assume four preferential positions. This is revealed by the pole distribution function (PDF) whose four maxima show that $c$-axes deviate from the ND by $26^{\circ}$ (M1), $16^{\circ}$ (M2), $38^{\circ}$ (M3), and $36^{\circ}$ (M4), respectively (see Fig. 4c). The identified orientations of $c$-axes reflect the microstructure division into regions in which grains start to grow from differently sloping surfaces. The four zones formed are depicted by means of axis/angle color coding (Fig. 4b). Additionally, they are also shown on the reconstructed surface of a rib (Fig. 4e). Details of the recreation, based on the EBSD data, are included in Appendix B. Each zone contains grains whose calcite $c$-axes orientate according to one of the four directions constituting the PDF maxima (the angular difference between them is not more than $10^{\circ}$ ). The three-dimensional reconstruction allows the distinct texture to be associated with the curved surface of the rib. It is worth noting that one of the slopes is disturbed. of grains is largely random. With shell development, low-energy boundaries are continued and others tend to disappear. This is shown by the results obtained for the cross-section (Fig. 3a and b). The surface image captures the area of elongated grains, whose longer boundaries are determined by the directions of growth. At the place of strong curvature in the growth line, they deviate from each other. This is enabled by the twin boundary that runs along the longitudinal direction (LD) (comp. Figs. 3d and 4a, b). In this way, the mirror symmetry between adjacent grains is combined with the symmetry of the entire area morphology.

The question arises as to how the microstructure induced by ribs affects the mechanical properties of the material. In order to solve the problem posed, research was carried out at different levels of the scale. In the first stage, static and dynamic nano-indentation of grains with various orientations was performed. Then, we conducted static compression tests of cubic elements representing rib areas with strongly inclined $c$-axes.

\section{Nano-indentation tests}

\subsection{Theoretical analysis}

Calcite anisotropy makes the orientation of prismatic grains a key determinant of global mechanical properties. The arrangement of these basic structural units controls the activation and course of plastic deformations, brittle fracture processes as well as their mutual coupling.

Experiments carried out for the geological single calcite crystal have identified two basic mechanisms of glide $r\{0 \overline{1} 14\}<02 \overline{2} 1>^{ \pm}$,

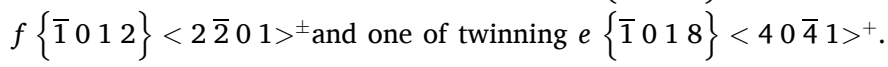
At room temperature, despite the high pressure caused by indentation, not all slip systems are activated, but only three out of six of each type: $r$ $<02 \overline{2} 1>^{-}, f<2 \overline{2} 01>^{-}$. Of course, this depends on the resolved shear stress operating in a given slip system. The lowest critical value of $110 \mathrm{MPa}$ was identified for the $r$ planes [40]. Hence $r<02 \overline{2} 1>^{-}$slips play a key role in the plastic deformation of calcite grains. The resolved shear stress can be determined by the Schmid factor $S$ according to the relationship: $\tau=\sigma_{0} S=\sigma_{0} \cos \varphi \cos \lambda$, where $\sigma_{0}$ is normal stress on the indented surface, while $\varphi$ and $\lambda$ are angles between the loading direction and the normal of a slip plane $\widehat{\boldsymbol{n}}$ or a slip direction $\widehat{\boldsymbol{s}}$, respectively. The Schmid factor depends on the orientation of a grain and the slip system that is activated inside it. If we assume an initial reference system with the $z$ axis parallel to the loading direction (see Fig. 5a) and a final one

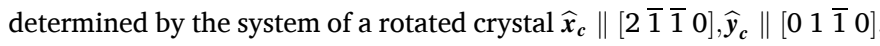
$\widehat{z}_{c} \|\left[\begin{array}{llll}0 & 0 & 0 & 1\end{array}\right]$ (Fig. 5), then the considered coefficient can be expressed by Euler angles and coordinates of unit vectors of the analyzed slip system $\widehat{n}$ and $\widehat{s}$. As a result, we obtain the following formula:

$S=\left(n_{x} \sin \varphi_{2} \sin \Phi+n_{y} \cos \varphi_{2} \sin \Phi+n_{z} \cos \Phi\right)\left(s_{x} \sin \varphi_{2} \sin \Phi+s_{y} \cos \varphi_{2} \sin \Phi+s_{z} \cos \Phi\right)$

Thus, the green zone located therein, characterized by the maximum M1, is divided into two parts. Apart from the distinguished areas, there are grains with $c$-axis orientations less preferred in the studied fragment. An example is a group of grains initiated in the rib ridge. Their $c$-axes remain subparallel to the ND.

Both calcite grains situated along the ND, as well as those inclined rotate around their $c$-axes, showing a tendency to locate the planes $\{01$ $-10\}$ perpendicular to the longitudinal direction (Fig. 4d). Actually, the rotation is continuous, so there are not clear angular preferences (Fig. 4e). We can say that, in the initial growth stage, the disorientation
The relation is depicted for those slip systems that give the highest values of the Schmid factor in the case of indented grains (Fig. 6a-c). A positive sign of the quantity means agreement between the signs of the applied normal stress and the resulting slip in the considered system.

Ionic bonding of calcite makes it prone to brittle fracture. The key

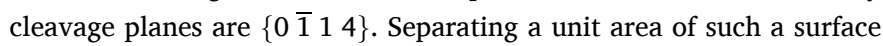
from the bulk crystal requires relatively little work, i.e. surface energy $\gamma_{C P}$. Subsequent cleavage planes with higher surface energy are

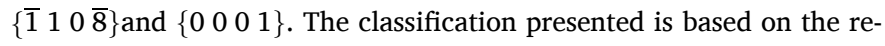



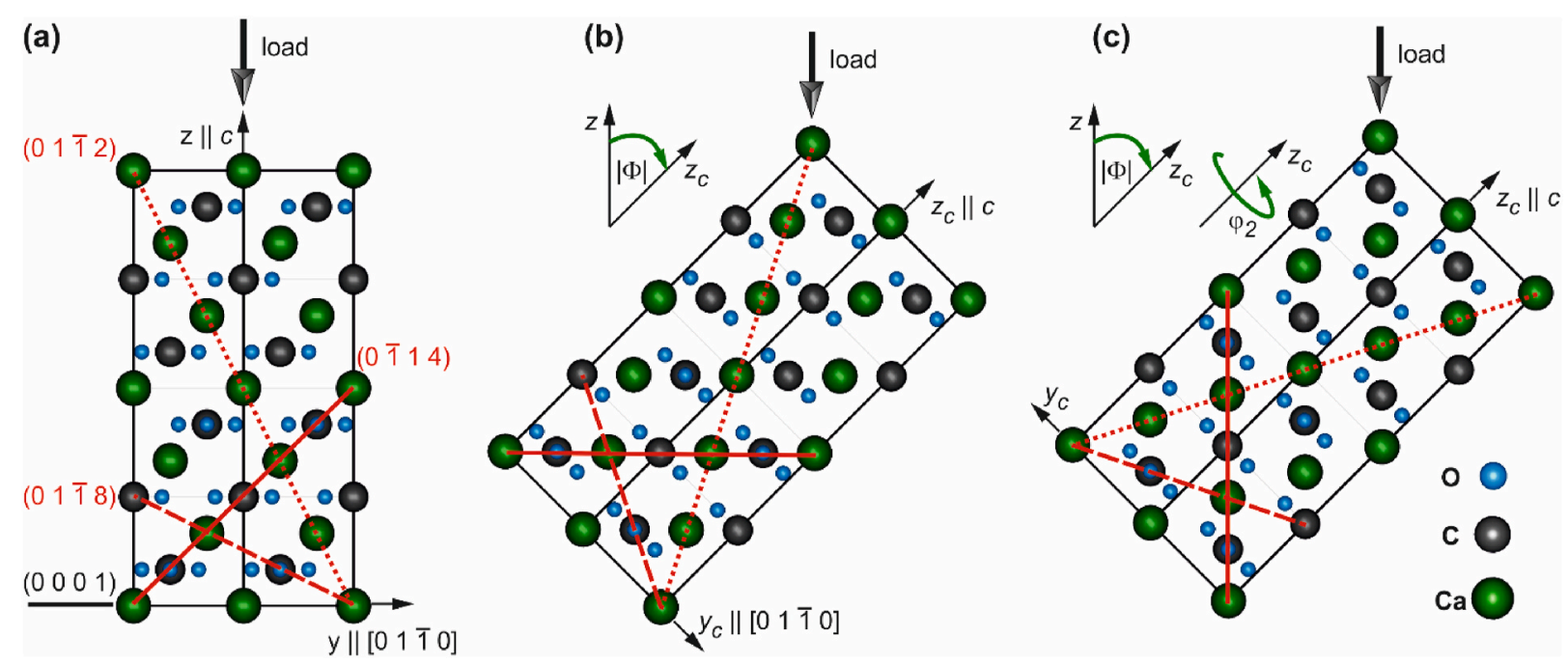

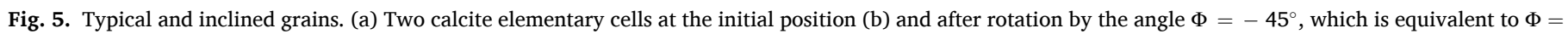

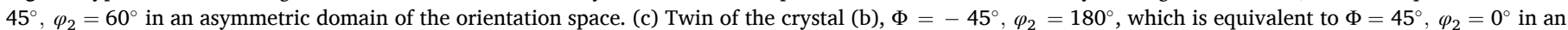
asymmetric domain. The planes for plastic and brittle deformation are also depicted.

sults obtained by Bruno et al. [41] using the molecular dynamics method. According to their calculations, the relaxed surface energy at 0 $\mathrm{K}$ for individual planes is $0.534,0.702$ and $0.711 \mathrm{~J} / \mathrm{m}^{2}$, respectively. Fracture in a given plane requires appropriate normal stresses. They arise during nano-indentations at the unloading stage. Their value can be calculated on the basis of simple geometrical relationships if we assume that there is a one-dimensional stress state $\sigma_{0}$ under the indenter: $\sigma=\sigma_{0} \cos ^{2} \varphi=\sigma_{0} C$. Thus, the amount of tensile stress on a given plane is determined by the cleavage factor $C$. Its value depends on coordinates of a unit vector normal to the plane and Euler angles that orientate an intended grain. This is expressed by the following formula:

$C=\left(n_{x} \sin \varphi_{2} \sin \Phi+n_{y} \cos \varphi_{2} \sin \Phi+n_{z} \cos \Phi\right)^{2}$

The relation is illustrated for three planes representing the mentioned families. The chosen planes generate the highest values of the factor in the case of intended grains (Fig. 6d-f).

Grains typical of the Pinna nobilis species with the $c$-axes running along the shell's thickness (ND) (Fig. 5a) are not the best mechanical solution. Then, regardless of the rotation of the grain around the $c$-axis, a maximal resolved shear stress equal to half the compressive stress applied to the outer surface is generated in the $\left(\begin{array}{llll}0 & \overline{1} & 1 & 4\end{array}\right)\left[\begin{array}{llll}0 & 2 & 2 & 1\end{array}\right]^{-}$system (Fig. 6a). Thus, in a given orientation of the grain, slips can be activated in three planes $\left\{\begin{array}{lll}0 & \overline{1} & 4\end{array}\right\}$ simultaneously. Similarly, basic twin systems $\left\{\begin{array}{lllll}1 & 0 & 1 & 8\end{array} 440 \overline{4} 1^{+}\right.$achieve a high Schmid factor exceeding 0.4 (Fig. 6b). They are activated during unloading. As a result, grains typical of Pinna nobilis undergo intensive plastic deformations. Moreover, there is a possibility of fracture along the $\left\{\begin{array}{lllll}0 & 0 & 0 & 1\end{array}\right\}$ plane because, during unloading, tensile stresses are perpendicular to it (Fig. 6f).

Geometric analysis of plastic processes shows that the inclination of grains significantly improves their mechanical properties. Due to the deviation of the $c$-axis from the outer surface normal, the systems of easy slips are so-oriented that the formation of shear stresses necessary to induce permanent deformations is difficult. As the angle Фincreases, the factor $S$ of the slip family $\left\{0 \begin{array}{lll}0 & \overline{1} & 1\end{array}\right\}<02 \overline{2} 1>$ decreases (Fig. 6a). Thus, during loading, the key glide mechanism $r<02 \overline{2} 1>^{-}$is confined.

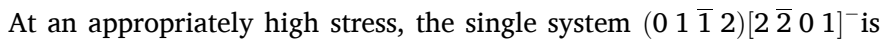
activated. Initially, it is constrained to a small group of grains with orientations near the $\left(49^{\circ}, 35^{\circ}\right)$ point, which constitutes the maximum Schmid factor (Fig. 6c). It is worth noting that, at the same time, in other systems of this family, the $S$ coefficient does not exceed 0.13 . At the unloading stage, the glide mechanism $r<02 \overline{2} 1>^{-}$is not activated at all. Instead, in the single systems $\left(\begin{array}{llll}\overline{1} & 0 & 1 & 8\end{array}\right)\left[\begin{array}{llll}4 & 0 & \overline{4} & 1\end{array}\right]^{+}$and

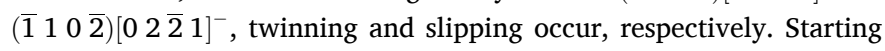
the last process is identified by the Schmid factor, which at the point $\left(71^{\circ}, 35^{\circ}\right)$ reaches the minimal value -0.5 .

Thus, with a relatively low external load, the glide processes inside typical grains are activated. When prismatic grains deviate from the ND above $30^{\circ}$, the initiation of slips requires the application of higher normal stresses to the outer surface. Then confined plastic deformations will occur in the systems $r<02 \overline{2} 1>^{-}$, and in grains with a specific

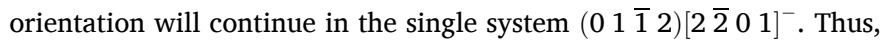
the degree of permanent deformation is lower. In the case of engineering materials, an increase in strength is associated with a decrease in fracture toughness. This relationship also affects the Pinna nobilis protective armor but is limited to a small group of grains with specific orientations determined by Euler's angles from the vicinity of the $\left(60^{\circ}, 45^{\circ}\right)$ point (Fig. 6d). Then the plane of easy cleavage (1 $01 \overline{1} 4)$ is located perpendicular to the normal force acting on the outer surface (see Fig. 5b). Hence, during unloading, an extensive crack will form. Other grains tend to split along the planes $\left\{\begin{array}{llll}\overline{1} & 1 & 0 & \overline{8}\end{array}\right\},\left\{\begin{array}{llll}0 & 0 & 0 & 1\end{array}\right\}$. Then, separation requires higher energy. Hence, the fracture process is initiated at a higher load and is often combined with plastic deformation, which limits the crack growth. To sum up, the anisotropy of calcite crystal makes the inclined prismatic grains show higher strength and fracture toughness in comparison to those with $c$-axes parallel to the ND. An exception is a small group in which the planes $\left\{\begin{array}{lll}1 & 0 & \overline{1}\end{array}\right\}$ orientate perpendicular to the ND. Nevertheless, the twin boundary $\left(\begin{array}{lll}0 & 1 & \overline{1}\end{array} 0\right)$ transforms the grains into the adjacent ones which show significantly higher strength and toughness (Fig. 5c).

\subsection{Experimental results}

The regularities revealed in the theoretical analysis are mapped by the relation that combines the values of the mechanical property 

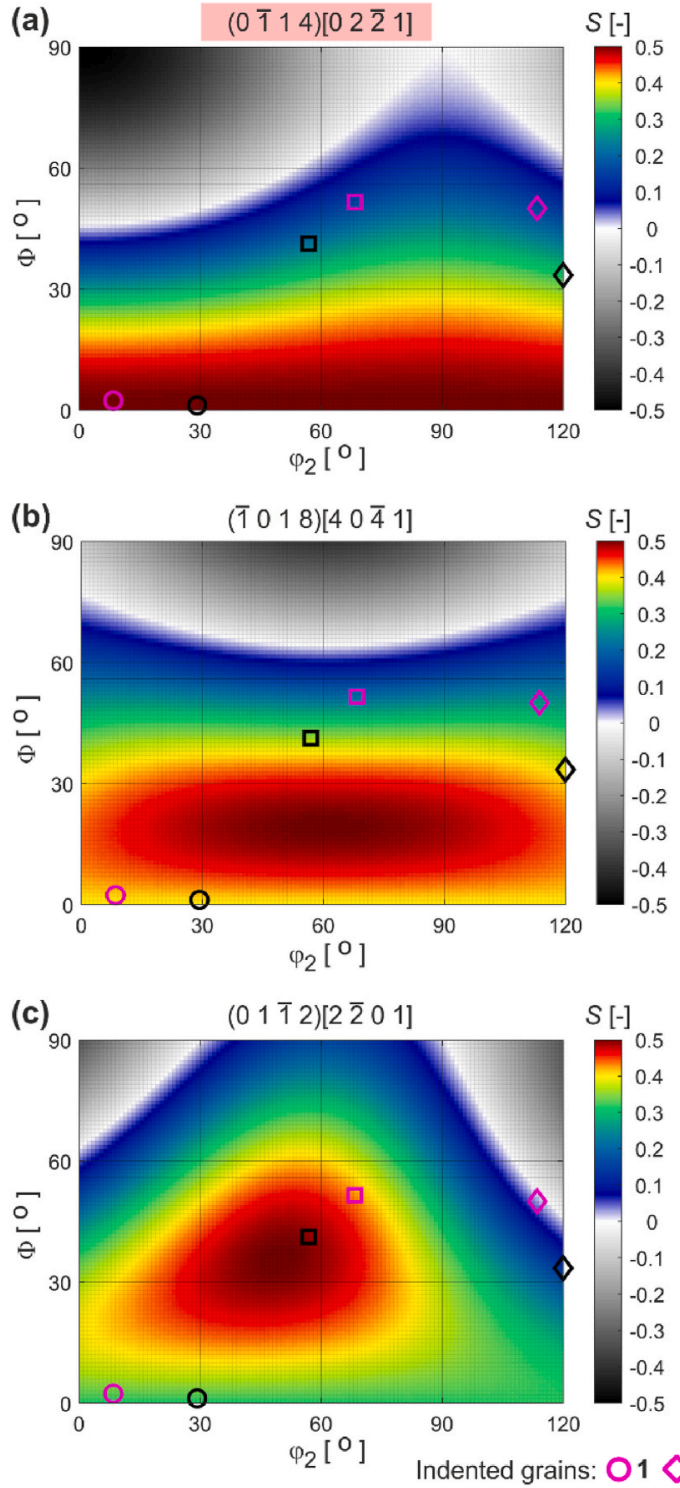

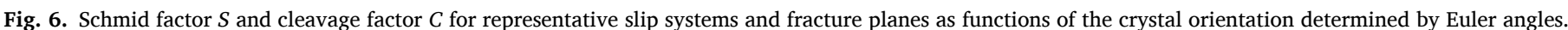

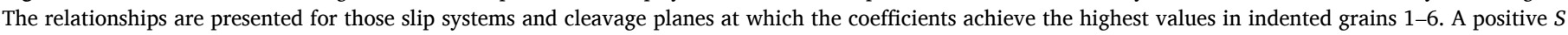
factor means that during unloading a positive slip arises, while at the loading stage a negative slip is generated.

Table 1

Mechanical properties of the shell's grains.

\begin{tabular}{llll}
\hline Grain & $\begin{array}{l}\text { Hardness } \\
{[\mathrm{GPa}]}\end{array}$ & $\begin{array}{l}\text { Reduced elastic modulus } \\
{[\mathrm{GPa}]}\end{array}$ & $\begin{array}{l}\text { Penetration depth } \\
{[\mathrm{nm}]}\end{array}$ \\
\hline 1 & $3.26 \pm 0.38$ & $66.8 \pm 4.8$ & $105.945 \pm 7.954$ \\
2 & $3.55 \pm 0.34$ & $66.4 \pm 4.2$ & $100.071 \pm 6.152$ \\
3 & $3.44 \pm 0.34$ & $75.1 \pm 5.3$ & $102.109 \pm 6.487$ \\
\hline
\end{tabular}

measured for representative grains in the nano-indentation test. Two groups were studied, each of which consists of three grains (see App. B). The first grain preserves the $c$-axis parallel to the ND, the second one shows a strong inclination, while the other due to the specific tilting has a plane $\left\{\begin{array}{lll}1 & 0 & \overline{1}\end{array} 4\right.$ \}oriented perpendicular to the ND with an accuracy of $10^{\circ}$. Schmid and cleavage factors for individual grains are presented in previously prepared distributions (Fig. 6). The first grain exhibits the
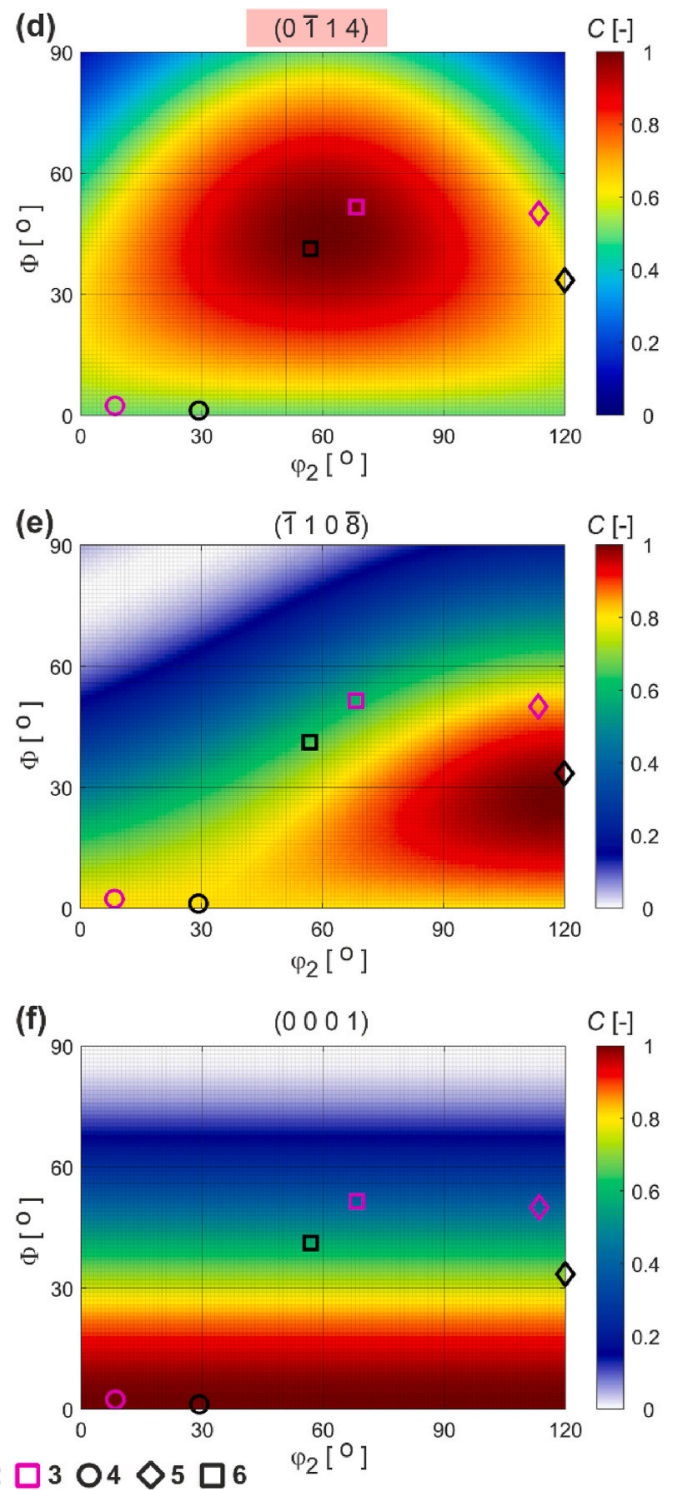


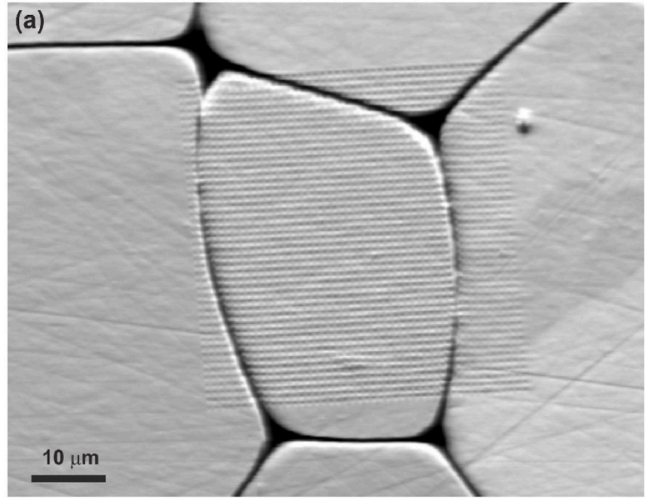

(c)

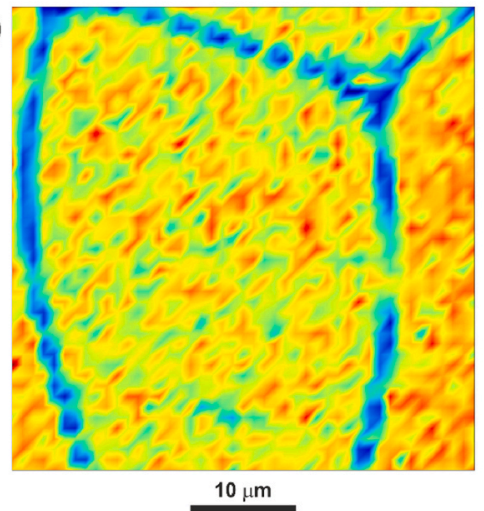

(e)

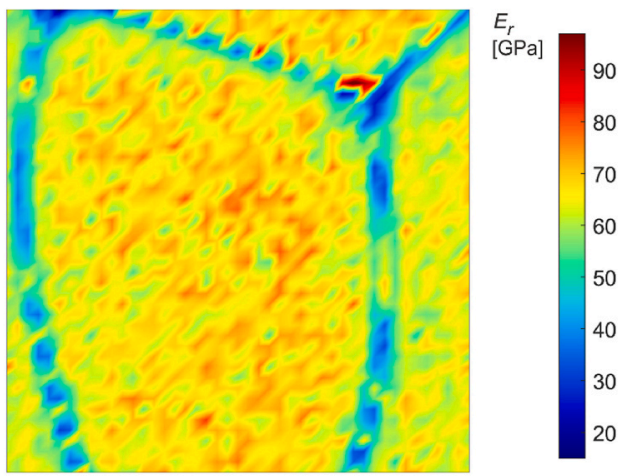

$\stackrel{H}{\text { [GPa] }}$

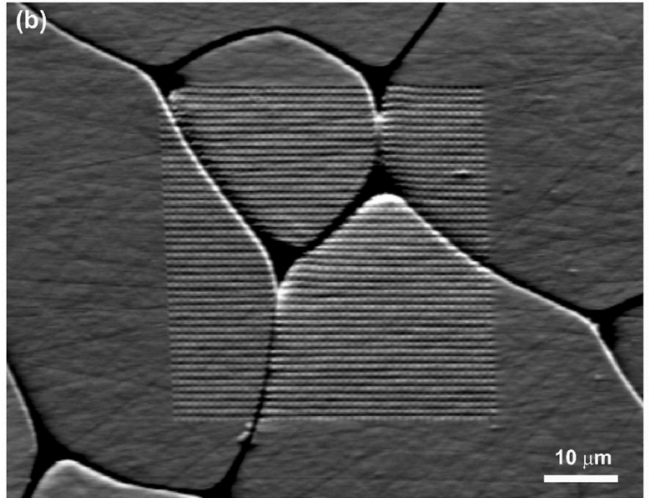

(d)

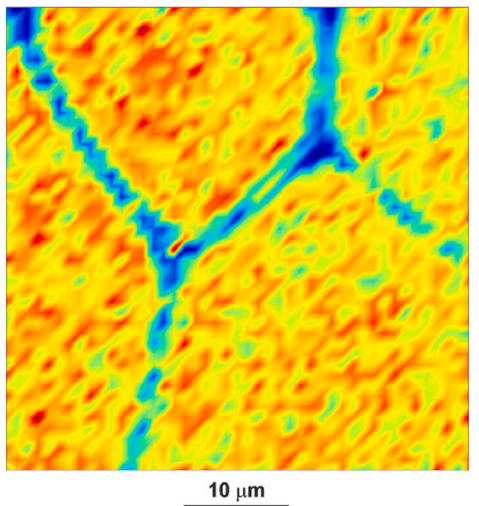

(f)

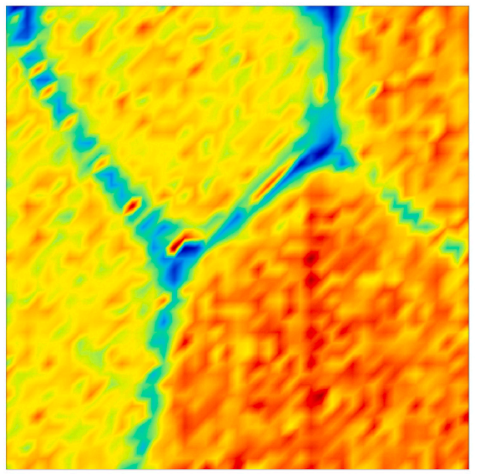

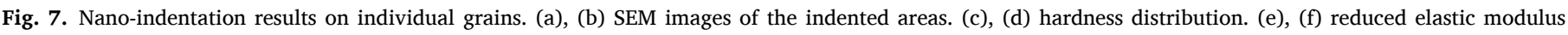
distribution.

disorientation, close to the twin relationship with respect to the plane $\left(\begin{array}{lll}0 & 1 & 1\end{array}\right)$, with the adjacent grain, number 2 . The rotation that occurs then causes the plane $\left(\begin{array}{lll}1 & 0 & \overline{1}\end{array}\right)$ to be almost vertical, i.e. parallel to the ND. As a result, the mechanical response is determined by a random plane with high indices. Its rare packing makes the elastic modulus the lowest. The orientations of grains 3 and 2 are similar to those shown in Fig. 5. The first of these grains exhibits the maximal cleavage factor for the key plane ( $\left.\begin{array}{llll}1 & 0 & \overline{1} & 4\end{array}\right)$. The other one, due to the disorientation close to the twin relationship, reduces this coefficient to 0 .

Additional information on how the orientation of the grains controls the mechanical properties is provided by the second group of grains subjected to the impact test (see App. B). Most cracks are formed in grain 4, with its $c$-axis subparallel to the ND, typical for Pinna nobilis shells (Fig. 8a). They develop in a radial direction from the corners of the impression or along the traces of planes $\left\{\begin{array}{llll}1 & 0 & \overline{1} & 4\end{array}\right\}$. The reason is significant plastic deformation caused at the loading stage. As in the case of the indentation of synthetic ceramics [42], the zone of plastic deformation located below the impression becomes a source of cracks that propagate to the external surface. According to theoretical analysis, a grain with the axis $c \|$ ND undergoes the strongest plastic deformation. This gives rise to a dense crack system. They combine to form surfaces along which portions of material adjacent to the indents' edges undergo separation. The inclined grain represented by grain 5 behaves completely differently (Fig. 8c). Individual cracks consistently run along the traces of the $(0 \overline{1} 14)$ plane deviated by an angle of $38^{\circ}$ from the surface (Fig. 8c). Thus, decohesion occurs at a lower depth, and in many cases is only partial. The atomic force microscopy (AFM) measurements show that the depths and areas of the impressions, as well as the heights of the material pileups, are smaller compared to grain 4 (compare 

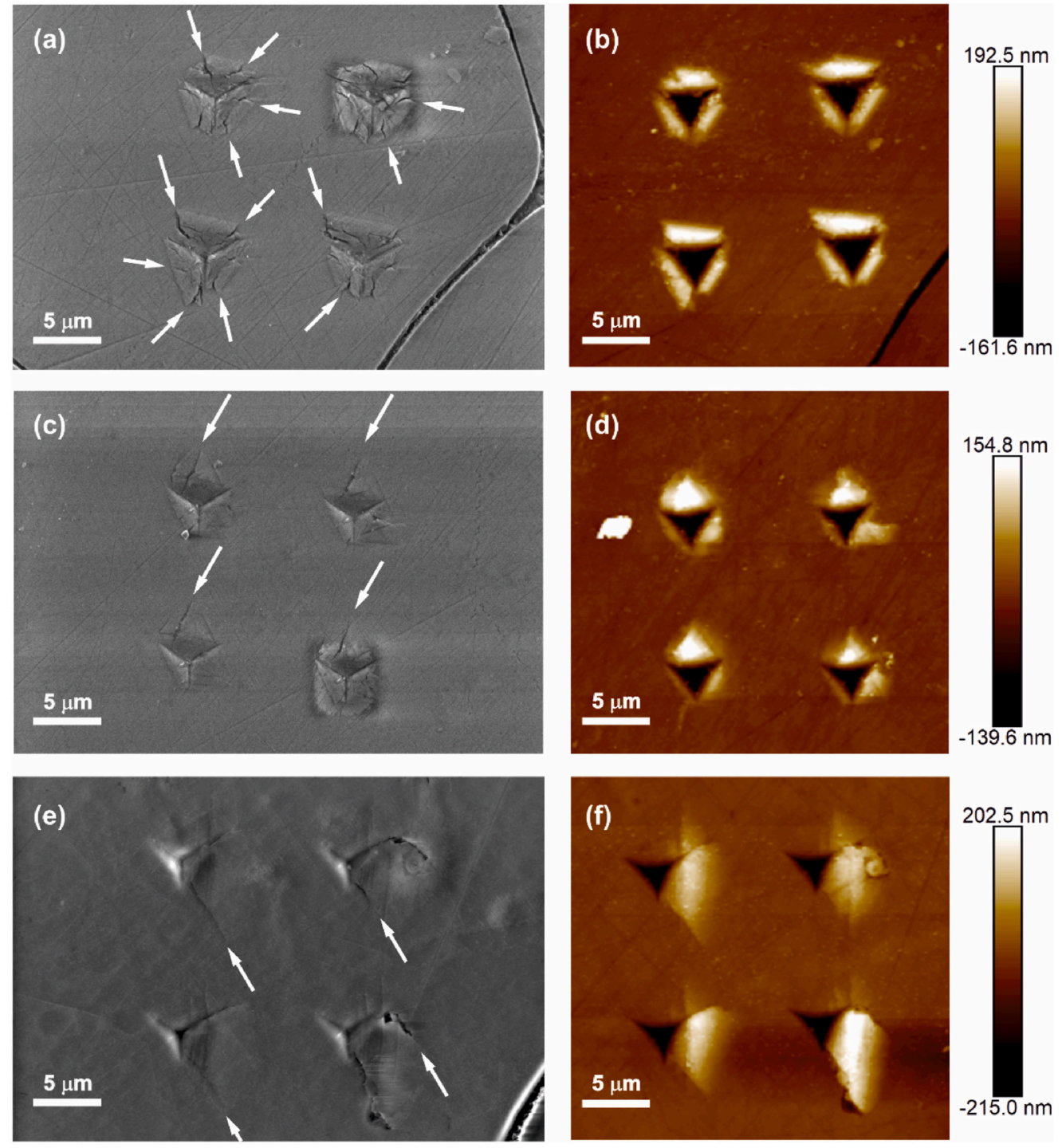

Fig. 8. Scanning electron microscopy (SEM) and atomic force microscopy (AFM) images of impressions in grains with different orientations. (a) (b) grain 4 with $c \|$

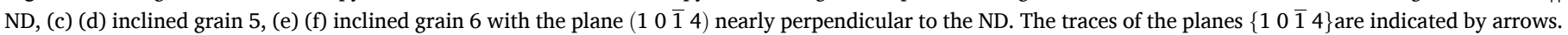
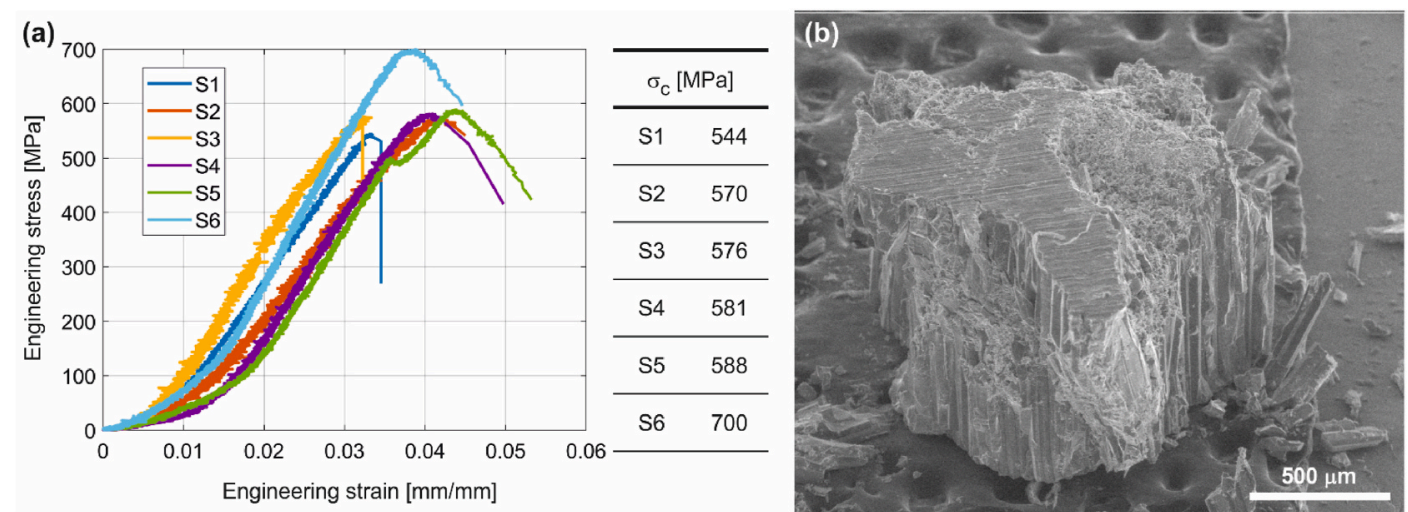

Fig. 9. Response of Pinna nobilis shell specimens to compression. (a) stress - strain curves together with compressive strength. (b) SEM image of specimen no. 5 after compression. 
Fig. 8 b and d). The visible reduction of plastic deformation, as well as limitation of fracture processes, is induced by the inclination of the prismatic grains. Higher strength and fracture toughness mean that absorption of the impact energy is much lower than in the case of grain 4. This is indicated by the greater distance to which the indenter is bounced from the sample back. It amounts to $4000 \mathrm{~nm}$ for grain 5, whereas for grain 4 it is $3800 \mathrm{~nm}$. A small group of oblique grains shows reduced fracture toughness. It is represented by grain 6 , which is illustrated in Fig. 6d. Then, during unloading, tensile stresses generate lateral cracks along the easy cleavage plane ( $\left.\begin{array}{llll}1 & 0 & \overline{1} & 4\end{array}\right)$ almost perpendicular to the ND. As a result, the material is chipped, which partly occurs along the plane $(1 \overline{1} 0 \overline{4})$ deviated by the large angle of $71^{\circ}$ from the surface (Fig. $8 \mathrm{e}$ and $\mathrm{f}$ ). Both the depths of impressions and the volumes of material detached from the bulk are the largest among the grains tested. Extensive damage entails significant absorption of impact energy, resulting in a shorter distance of the indenter bounce $-3600 \mathrm{~nm}$.

\section{Compression tests}

Besides the identification of mechanical properties at the nano- and microscopic levels, the response at the macroscopic level was also examined. A compression test was carried out for 6 samples cut from the rib areas of the CCP layer at the places of scales (see Fig. 4b) where the grains strongly deviate from the ND. The results, in the form of the stress-strain diagram together with the compressive strengths $\left(\sigma_{c}\right)$ determined for the individual shell specimens, are presented in Fig. 9.

The values obtained are remarkably high considering the mechanical properties of the materials the shell is made of. Compressive strength up to $700 \mathrm{MPa}$ results from the unique structure which is a weave of strong and weak units. This gives a great ability to dissipate energy and also enables blocking and delocalization of the fracture process. As a result, the loss of load capacity occurs at high stresses, when a significant part of the structure or even the entire structure is destroyed. Examples of such mechanical responses are samples 5 and 6, respectively.

\section{Conclusions}

The present work focuses on radial ribs in a shell of the Pinna nobilis species. The external part of this protective armor is built of calcite prismatic grains running perpendicular to the outer surface. The rib area is, naturally, strongly curved. Thus, grains starting the growth orientate obliquely to the thickness direction. Angles between the $c$-axes of calcite grains and the ND are considerable and often exceed $30^{\circ}$. With development, the structure is ordered and the prismatic grains reach the nacre layer perpendicularly. Despite this, the grain crystallographic $c$-axes preserve their initial oblique orientation. The EBSD investigations reveal that the disorientation of grains is not random. Most often they rotate relative to each other around the $c$-axis by $18^{\circ}, 38^{\circ}$ and $60^{\circ}$. In order to uncover the reason for the observed strong preference, the interface energy change during the continuous rotation of one grain relative to the adjacent one was calculated. The results obtained show that neighboring grains form low-energy twin disorientations. Thanks to them, a dominant $c$-axis orientation arises at the outer surface of the shell and is then transferred deeper up to the nacre layer. The twin boundary

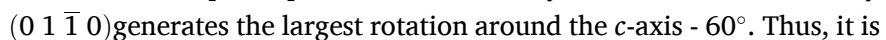
often located along the symmetry axis of the shell rib enabling strong curvature of the growth line while maintaining coherency of the areas on the left and right.

In the flat predominant areas of the shell, grains typical of $P$. nobilis are formed, with the calcite $c$-axes subparallel to the ND. Such an orientation means that the load applied perpendicular to the outer surface induces significant resolved shear stresses in the calcite easy slip planes $\left\{\begin{array}{llll}1 & 0 & \overline{1} & 4\end{array}\right\}$. This leads to intense plastic deformations and, as a result, to lowered hardness, confirmed in the nano-indentation tests. The zone of strong plastic strains formed during loading becomes the source of the network of cracks combining into the cleavage planes at the unloading stage. Thus, the typical grains are also characterized by the reduced fracture toughness, as shown in the nano-impact tests.

The recipe for improving the mechanical properties is the inclination of the calcite $c$-axis relative to the ND. This was demonstrated in the theoretical analysis by determining the distributions of the Schmid and cleavage factors. A favorable oblique $c$-axis orientation is generated in the slopes of ribs. Nano-indentation and nano-impact tests performed on representative grains of this zone confirm higher hardness and fracture toughness.

Samples from rib areas with strongly tilted $c$-axes were subjected to a compression test. Their strength is surprisingly high - reaching $700 \mathrm{MPa}$. The excellent mechanical properties of the biogenic material result from the weave of strong and weak structural units. The former provides load transfer, while the latter constitutes zones of controlled energy dissipation, which is necessary to reduce brittle fracture processes.

In summary, well-shaped ribs act as a shell's reinforcement. Their unique mechanical properties are generated by prismatic grains with $c$ axes strongly deviated from the thickness direction. They exhibit high strength and fracture toughness. The grains of the special orientation and their role in load transfer is revealed here for the first time.

\section{Materials and methods}

Microstructure investigations. The $P$. nobilis specimen was collected in 2013 from the bay of Almyropotamos, Evia island, Central Greece. The species is strongly protected by a European Council Directive (92/43/CEE). According to Article 16 paragraph 1(d) of the Directive, the specimen was collected for the purpose of scientific research. The samples were cut along both the ribs and directions perpendicular to them so as to obtain cuboids with their bases being the outer and inner shell surfaces. The obtained blocks were separately embedded in epoxy resin. The samples were ground and polished according to standard metallographic preparation protocols: mechanical polishing with silicon carbide papers 220-7000 grit followed by $1 \mu \mathrm{m}$ and $0.25 \mu \mathrm{m}$ diamond suspension and a final polishing step with colloidal silica suspension for 5 min using a Struers Tegramin-25 automatic polisher. The morphology and the surface characterization of the shell were studied with a Keyence VHX-7000 digital optical microscope and a contact profilometer (DektakXT Bruker Stylus Profiler, Billerica, USA) equipped with a diamond microneedle. Roughness was measured in a representative rib area of $0.5 \times 0.5 \mathrm{~mm}$. The arithmetic average roughness in the longitudinal and transverse directions was determined by decomposing the appropriate profiles with the use of cubic splines. EBSD analyses were carried out using an FEI Versa 3D scanning electron microscope (SEM) equipped with an EDAX Hikari CCD camera. Diffraction patterns were collected under low vacuum $\left(30 \mathrm{~Pa}\right.$ of $\left.\mathrm{H}_{2} \mathrm{O}\right)$ at accelerating voltage $(15 \mathrm{kV})$. The obtained EBSD data were analyzed using the MTEX software [43].

Disorientation. It unequivocally defines an orientation relationship between crystallites. The disorientation is determined by the smallest angle of rotation around an axis, which transforms the reference system of one crystal into another one. Due to symmetry equivalence, a given orientation relationship between crystals can be expressed by many different angle/axis pairs which constitute a set of misorientations. 
Estimation of interfacial energy. According to the Gautam - Howe method, the overlapping intensity $I$ is a measure of the bonding strength at the phase boundary i.e. of matching the potential fields. Thus the higher the quantity $I$, the lower the energy of the interface treated as a disturbance [44]. In order to determine the distribution of total over-

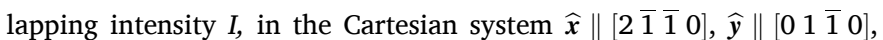
$\widehat{z} \|\left(\begin{array}{llll}0 & 0 & 0 & 1\end{array}\right)$, two calcite crystals are considered. One of them is fixed, while the other rotates around the axis $z$ by the angle $\omega$. The antisymmetric domain is investigated with an accuracy of $0.1^{\circ}$, thus $\omega \in<0^{\circ}$, $60^{\circ}>$. Diffraction intensity $I_{i}$ was assigned to each node in the reciprocal space using the structure factor $I_{i}=F_{i} \cdot F_{i}^{*}[45,46]$. It is assumed that the intensity is distributed according to the Lorentzian function $s_{i}(r)=$ $\frac{I_{i} \Gamma_{i}}{2 \pi^{2} r^{2}\left(r^{2}+\Gamma_{i}^{2}\right)}$, where $\Gamma_{i}$ is half of the width at half of the maximum. $\Gamma_{i}$ constitutes the part of the sphere radius $R_{i}$ bounding a reflection. With the appropriate relation $R_{i} / \Gamma_{i}$, the intensity contained in the sphere $I_{c i}$ is almost all of the intensity $I_{i}$ assigned to a given node: $I_{c i}=\int_{0}^{R_{i}} \int_{0}^{2 \pi} \int_{0}^{\pi}$ $s_{i}(r) r^{2} \sin \varphi \mathrm{d} \varphi \mathrm{d} \theta \mathrm{d} r=\frac{2}{\pi} \arctan \left(\frac{R_{i}}{\Gamma_{i}}\right)$. It is assumed that $\Gamma_{i}=\frac{R_{i}}{12}$, then nearly $95 \%$ of the intensity $I_{i}$ is taken into account. The distribution of diffraction intensities in the reciprocal space maps the potential field of a crystal. The introduction of a phase boundary significantly disturbs the continuity of the field along a given family of planes if there is a considerable misfit between the systems of the planes in the neighboring crystallites. Thus, it is assumed that the bonding along the most densely packed planes of calcite $\left(\begin{array}{lll}1 & 0 & \overline{1}\end{array}\right)$ is broken when the mismatch amounts to $20 \%$. As a result, using formulas derived in Ref. [44], radii of the individual spheres are obtained as $R_{i}=\frac{0.1 I_{i}}{d_{1} I_{1}}$, while $d_{1}$ and $I_{1}$ are interplanar distance and intensity for the considered system of planes, respectively.

Identification of mechanical properties. Nano-indentation and nano-impact tests were carried out parallel to the shell thickness direction by means of the NanoTest Vantage system with a diamond Berkovich three-sided pyramid indenter. Indentations were made in the "load control" mode with 1-s loading time, 1-s dwell at a maximal force of $1.5 \mathrm{mN}$ and a half-second unloading time. Thanks to high-speed tests, grids of 2025 impressions, $1 \mu \mathrm{m}$ apart, were performed in two regions. They became the basis for determining the maps of the hardness and reduced Young's modulus. The first region contains the studied G1 grain, while the second one - further considered G2 and G3 grains. In order to obtain the mechanical characteristics in the areas of the studied grains, appropriate SEM images were superimposed on the maps. In this way, 3 groups of imprints were separated. Each chosen impression is located inside one of the considered grains at a distance of not less than $1 \mu \mathrm{m}$ from the boundary. As a result, the average values of $H$ and $E_{r}$ for G1, G2 and G3 were determined, eliminating the influence of voids surrounding grains. The Nano Tests instruments also open the possibility to conduct an impact test [47]. The Berkovich indenter was used, which was moved away from the fixed specimen to obtain an accelerating force of $0.5 \mathrm{mN}$. The amplitude of the first rebound from the sample surface was measured, which gave information about the amount of energy dissipated in the material. Atomic force microscopy (AFM) measurements were determined with a Bruker Dimension ICON XR operating in the PeakForce-QNM mode in air. Data analysis was performed using NanoScope Analysis software. At the last stage of research, the static compression test was carried out. An MTS 810 servo-hydraulic test machine, equipped with an additional $25 \mathrm{kN}$ load cell and an MTS clip gauge extensometer, was used. The specimens were cut from the shell using diamantine wire and had the geometry of a rectangular prism with an average cross-sectional dimension of $2 \mathrm{~mm}$ and an average height (thickness) of $1.2 \mathrm{~mm}$. The samples were compressed in the direction of the shell thickness under grip displacement control at a speed of 0.2 $\mu \mathrm{m} / \mathrm{s}$.

\section{CRediT authorship contribution statement}

Kinga Nalepka: Conceptualization, Investigation, Formal analysis, Calculations constituting the basis for the theoretical analysis of microstructure and mechanical properties, Writing - original draft, Writing - Review \& Editing. Katarzyna Berent: Conceptualization, Investigation, Formal analysis, SEM/EBSD measurements, Writing original draft, Writing - Review \& Editing. Antonio G. Checa: Conceptualization , Methodology, Writing - original draft, Writing Review \& Editing. Tomasz Machniewicz: Investigation, Formal analysis, Mechanical measurements, Writing - original draft, Writing - Review \& Editing. Adrian J. Harris: Mechanical measurements, Formal analysis. Pawel Nalepka: Formal analysis, Investigation. Martyna Strąg: Resources, Visualization. Lukasz Maj: Data Curation, Resources. Aleksandra Szkudlarek: Investigation, AFM measurements. Krzysztof Sztwiertnia: Methodology, Supervision, Funding acquisition.

\section{Declaration of competing interest}

The authors declare that they have no known competing financial interests or personal relationships that could have appeared to influence the work reported in this paper.

\section{Acknowledgments}

The authors thank Mr Łukasz Niedzielski from Keyence International for the possibility of performing microstructural analysis using a VHX7000 digital microscope. Thanks to Damian Sapijaszka who collected the $P$. nobilis specimen for scientific research. The work was supported by the Polish National Agency for Academic Exchange (grant PPI/APM/ 2018/1/00049/U/001) and the National Science Center (grant UMO2018/29/B/ST8/02200). MS was supported by the European Union from the resources of the European Social Fund (Project No.WNDPOWR.03.02.00-00-I043/16). AGC was funded by project CGL201785118-P of the Spanish Ministerio de Ciencia e Innovación. José Rafael García March (Universidad Católica de Valencia, Spain) provided the images of Pinna nobilis of Fig. A1.

\section{Appendix A. Morphology of a typical Pinna nobilis shell}

The appearance of the examined shell, which was broken at the early stage of growth, differs insignificantly from the shell representative for the $P$. nobilis species. In order to facilitate the comparison, additional images of the external and internal surfaces of a typical specimen were created (Fig. A.1). The ribs run uninterruptedly from the narrow umbo to the broad posterior margin, are quite distinct and create a characteristic sculpture of the shell. The only difference is in the part of the posterior margin, which in this case is one whole. Nacre occupies most of the anterior half of the shell and is divided into two lobes by a narrow longitudinal sulcus. The middle part of the shell, in which the median ridge (ligament) is located, has a similar structure in both cases. The surface of the sample is characterized by large, widely spaced spines and rows of scales. Pinnoidea live partially buried to one third of their length in sediment [48]. Therefore, part of the spines on the surface can be abraded and absent in the anterior portion of the shell. This is particularly evident in adult specimens. Undamaged $P$. nobilis has scars that are formed during the degradation of the spines. The surface ribbing is similar to the damaged specimen studied in the present work. 


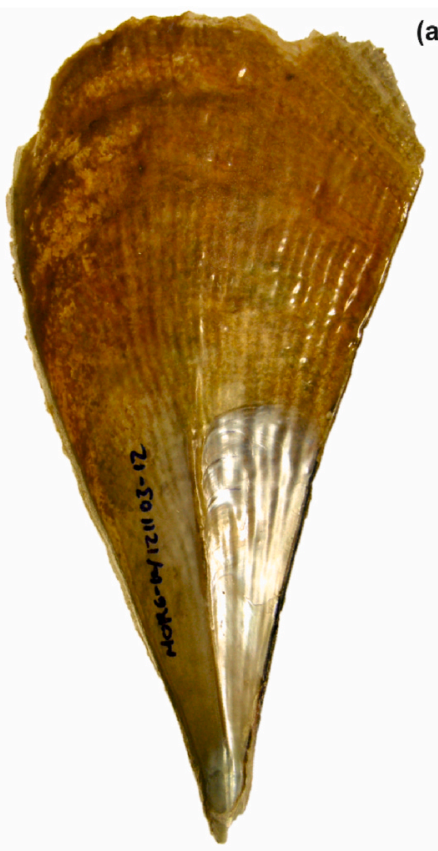

(a)

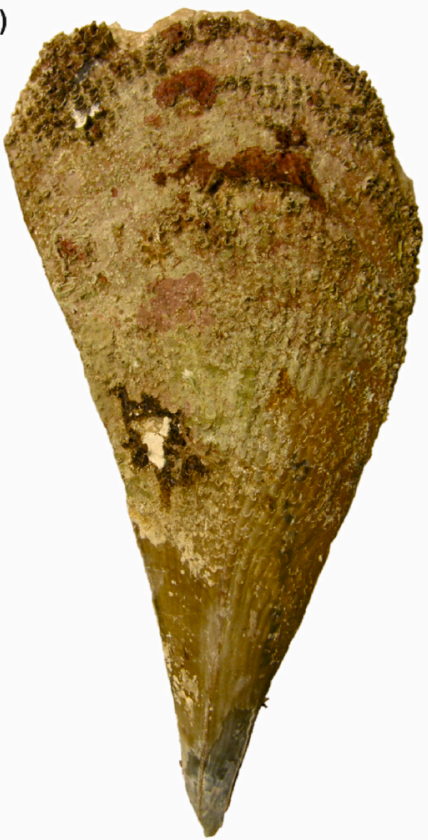

$5 \mathrm{~cm}$

(b)

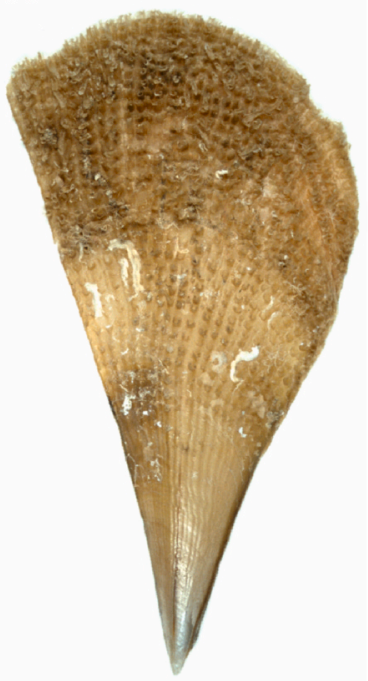

Fig. A.1. Views of typical P. nobilis shells. (a) Internal and external views of the left valve of an adult specimen. (b) External view of the left valve of a submature shell..

\section{Appendix B. Arrangement of grains subjected to nano-indentation and impact tests}

The examinations were carried out in the area of the rib belonging to the posterior of the primary shell, below the fracture trace (see Fig. B.1). Basic structural units, prismatic grains, grow perpendicular to the outer surface. Thus, the calcite $c$-axes of the grains runs along the radii of the rib curvature.

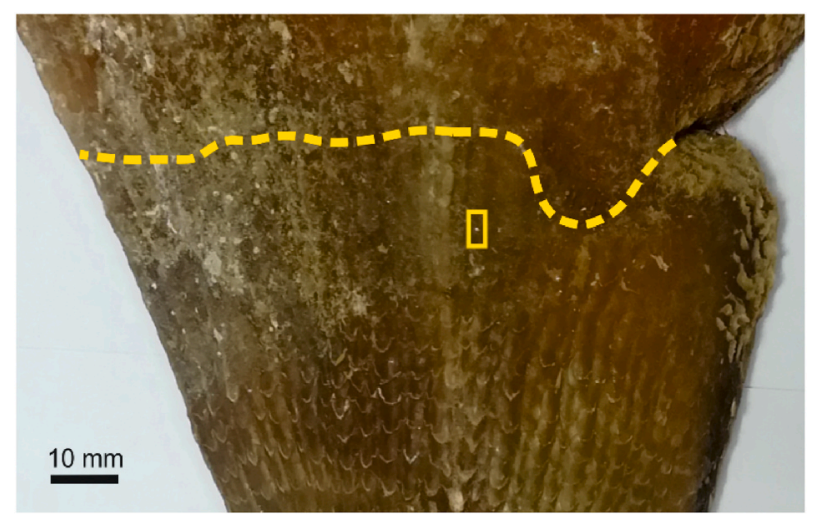

Fig. B.1. The location of the area in which nano-indentation and impact tests were conducted. The tested region (yellow rectangle) is in the ventral part of the left valve, below the fracture trace marked by broken yellow line.

The nano-indentation test was performed on three grains with specific orientations (see Fig. B.2 a). The $c$-axis of the first one (G1) remains parallel to the ND with an accuracy of $2^{\circ}$ and in the next two (G2, G3) it deviates by an angle of $50^{\circ}$. Between the G2 and G3 grains there is disorientation close to the twin relationship with respect to the plane $\left(\begin{array}{llll}1 & 0 & 1 & 0\end{array}\right)$. Thus, the normal to the easy cleavage and slip plane $\left(\begin{array}{llll}1 & 0 & \overline{1} & 4\end{array}\right)$, deviated by an angle of $39^{\circ}$ from the ND in G2, undergoes rotation and forms a small angle of $9^{\circ}$ with the ND. Consequently, the G2 grain shows a high fracture toughness and the G3 grain significantly lower. The first grain G1 was initiated in the ridge of the rib (see Fig. B.2 b). As a result, the $c$-axis subparallel to the ND exhibits the orientation typical of $P$. nobilis species, arising in the prevalent flat areas of the shell. The two subsequent grains start growth in the slope of the rib. This leads to the oblique $c$-axis orientation required for units with high hardness and fracture toughness. The geometry of the outer rib surface (see Fig. B.2 b) was reconstructed on the basis of the EBSD map, where the orientations of the $c$-axes in the subsequent points of the shell were recorded with the step of $1.6 \mu \mathrm{m}$. A similar procedure was applied to the area subjected to the impact test (see Fig. B. 3 a). The obtained outer surface shows that the dynamically loaded grains G4, G5 and G6 are located analogously to G1, G2 and G3 (see Fig. B.3 b). As a result, the $c$-axis of the G4 grain is almost parallel to the ND, the deflection angle is $1^{\circ}$. Grains G5 and G6 orientate obliquely, the angles between the ND and $c$-axes amount to $32^{\circ}$ and $42^{\circ}$, 
respectively. The first of them shows high fracture toughness, while the other one is significantly lower due to the location of the plane (1 $0 \overline{1} 4)$ nearly parallel to the outer surface, the deviation angle is $4^{\circ}$.

In the areas of mechanical investigations, the average grain sizes amount to $45.118 \mu \mathrm{m} \pm 26.619 \mu \mathrm{m}$ (Fig. B.3a) and $45.044 \mu \mathrm{m} \pm 18.071 \mu \mathrm{m}$ (Fig. B.3a). The high value of the standard deviation indicates considerable diversity, which is also represented by the set of grains subjected to the nano-indentation (see Fig. B.3a) and nano-impact tests (Fig. B.3a). This feature characterizes the layers close to the outer surface of the shell from which the growth of prismatic structural units begins.

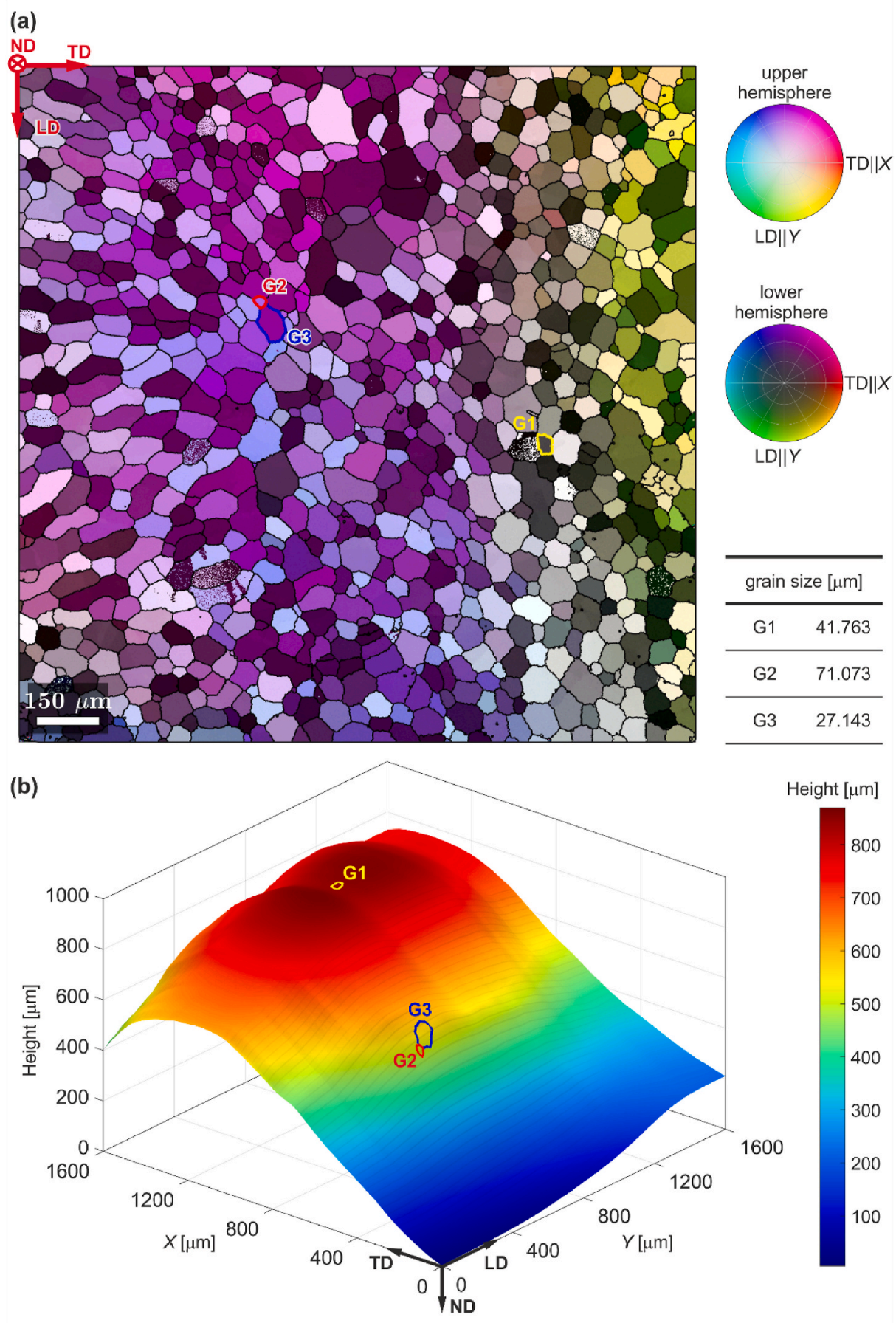

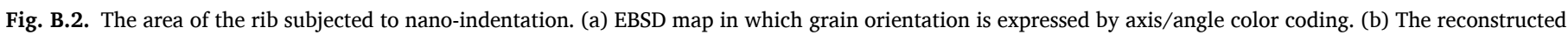
geometry of the outer surface. The boundaries of the intended grains G1, G2 and G3 (see Fig. 7) are marked in yellow, red and blue, respectively. 
(a)
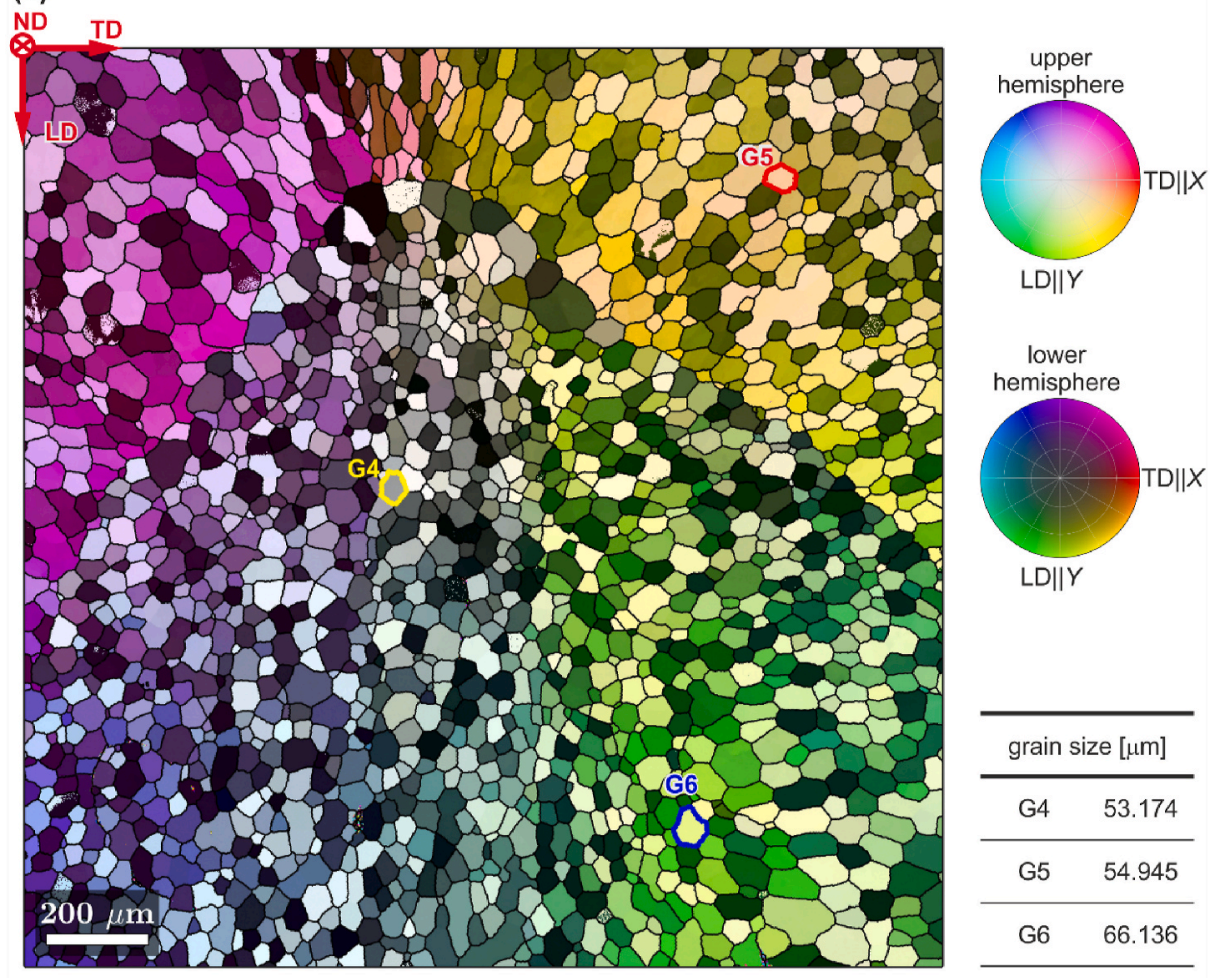

(b)

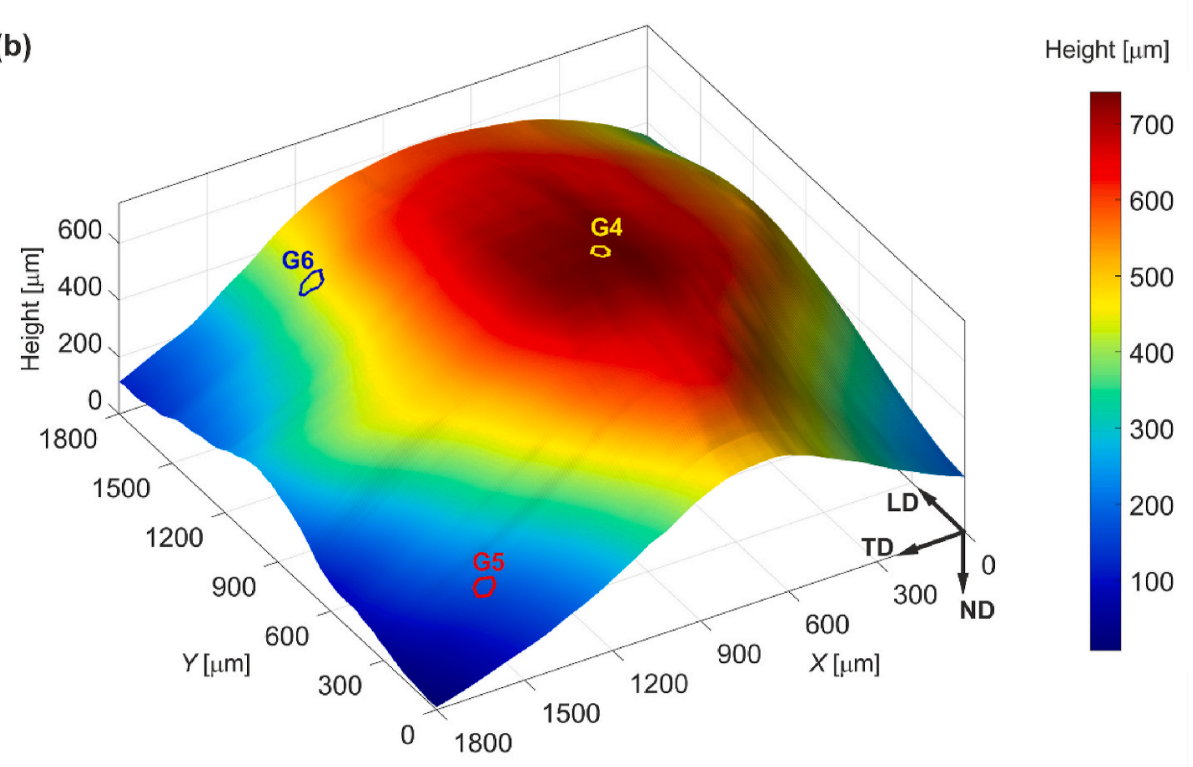

Fig. B.3. The area of the rib subjected to the nano-impact test. (a) EBSD map in which grain orientation is expressed by axis/angle color coding. (b) The reconstructed geometry of the outer surface. The boundaries of the impacted grains G4, G5 and G6 (see Fig. 8) are marked in yellow, red and blue, respectively.

\section{References}

[1] A.G. Checa, A.B. Rodríguez-Navarro, F.J. Esteban-Delgado, The nature and formation of calcitic columnar prismatic shell layers in pteriomorphian bivalves, Biomaterials 26 (2005) 6404-6414, https://doi.org/10.1016/j. biomaterials.2005.04.016.

[2] J.S. Bowerbank, On the structure of the shells of molluscous and conchiferous animals, Trans. Microsc. Soc. Lond. 1 (1844) 123-154.

[3] O.B. Böggild, The shell structure of the mollusks. Det K. Danske Vidensk. Selsk. Skr, Naturvidenskabelig og Math. Afd. Raekke 92 (1930) 231-326.

[4] D. Volkmer, M. Harms, L. Gower, A. Ziegler, Morphosynthesis of nacre-type laminated $\mathrm{CaCO}_{3}$ thin films and coatings, Angew. Chem. Int. Ed. 44 (2005) 639-644, https://doi.org/10.1002/anie.200461386.
[5] A. Finnemore, P. Cunha, T. Shean, S. Vignolini, S. Guldin, M. Oyen, U. Steiner, Biomimetic layer-by-layer assembly of artificial nacre, Nat. Commun. 3 (2012) 1-6, https://doi.org/10.1038/ncomms1970.

[6] Ch Xiao, M. Li, B. Wang, M.-F. Liu, Ch Shao, H. Pan, Y. Lu, B.-B. Xu, S. Li, D. Zhan, Y. Jiang, R. Tang, X.Y. Liu, H. Cölfen, Total morphosynthesis of biomimetic prismatic-type $\mathrm{CaCO}_{3}$ thin films, Nat. Commun. 8 (2017) 1-9, https://doi.org/ 10.1038/s41467-017-01719-6.

[7] H. Zhao, Z. Yang, L. Guo, Nacre-inspired composites with different macroscopic dimensions: strategies for improved mechanical performance and applications, NPG Asia Mater. 10 (2018) 1-22, https://doi.org/10.1038/s41427-018-0009-6.

[8] S. Schenk, I. Zlotnikov, B. Pokroy, N. Gierlinger, A. Masic, P. Zaslansky, A.N. Fitch, O. Paris, T.H. Metzger, H. Cölfen, P. Fratzl, B. Aichmayer, Hierarchical calcite crystals with occlusions of a simple polyelectrolyte mimic complex biomineral 
structures, Adv. Funct. Mater. 22 (2012) 4668-4676, https://doi.org/10.1002/ adfm. 201201158.

[9] T.H. Metzger, Y. Politi, G. Carbone, B. Bayerlein, I. Zlotnikov, E. Zolotoyabko, P. Fratzl, Nanostructure of biogenic calcite and its modification under annealing: study by high-resolution X-ray diffraction and nanoindentation, Cryst. Growth Des. 14 (2014) 5275-5282, https://doi.org/10.1021/cg501068e.

[10] S. Lemer, V.L. González, R. Bieler, G. Giribet, Cementing mussels to oysters in the pteriomorphia tree: a phylogenomic approach, Proc. R. Soc. B Biol. Sci. 283 (2016), 20160857, https://doi.org/10.1098/rspb.2016.0857.

[11] J.D. Taylor, W.J. Kennedy, A. Hall, The shell structure and mineralogy of the Bivalvia: Introduction, Nuculacea-Trigonacea. Bull. Br. Mus. (Nat. Hist.) Zool., Lond. (Supplement 3) (1969) 1-125.

[12] J.R. García-March, A.M. García-Carrascosa, A.L. Peña Cantero, Y.G. Wang, Population structure, mortality and growth of Pinna nobilis Linnaeus, 1758 (Mollusca, Bivalvia) at different depths in Moraira bay (Alicante, Western Mediterranean), Mar. Biol. 150 (2007) 861-871, https://doi.org/10.1007/s00227 006-0386-1.

[13] D. Zavodnik, M. Hrs-Brenko, M. Legac, Synopsis on the fan shell Pinna nobilis L. in the eastern Adriatic Sea, in: C.F. Boudouresque, M. Avon, V. Gravez (Eds.), Les espèces Mar. à protéger en Méditerranée. Marseille, GIS Posidonie publications, Marseille, France, 1991, pp. 169-178.

[14] Y. Dauphin, Soluble organic matrices of the calcitic prismatic shell layers of two pteriomorphid bivalves: Pinna nobilis and Pinctada margaritifera, J. Biol. Chem. 278 (2003) 15168-15177, https://doi.org/10.1074/jbc.M204375200.

[15] E.M. Harper, A.G. Checa, Tightly shut: flexible valve margins and microstructural asymmetry in pterioid bivalves, Mar. Biol. 167 (2020) 1-12, https://doi.org/ 10.1007/s00227-020-03693-y.

[16] F. Marin, P. Narayanappa, S. Motreuil, Acidic shell proteins of the Mediterranean fan mussel Pinna nobilis - molecular Biomineralization, in: W. Müller (Ed.), Prog. Mol. Subcell. Biol. 52 (2011) 353-395, https://doi.org/10.1007/978-3-64221230-7_13. Springer, Berlin, Heidelberg.

[17] E.M. Harper, A. Checa, Physiological versus biological control in bivalve calcite prisms: comparison of Euheterodonts and Pteriomorphs, Biol. Bull. 232 (2017) 19-29, https://doi.org/10.1086/691382.

[18] Y. Dauphin, Comparison of the soluble matrices of the calcitic prismatic layer of Pinna nobilis (Mollusca, Bivalvia, Pteriomorpha), Comp. Biochem. Physiol. Mol. Integr. Physiol. 132 (2002) 577-590, https://doi.org/10.1016/s1095-6433(02) 00099-5.

[19] A.G. Checa, J.T. Bonarski, M.G. Willinger, M. Faryna, K. Berent, B. Kania, A. González-Segura, C.M. Pina, J. Pospiech, A. Morawiec, Crystallographic orientation inhomogeneity and crystal splitting in biogenic calcite, J. R. Soc. Interface 10 (2013), 20130425, https://doi.org/10.1098/rsif.2013.0425.

[20] L. Addadi, Y. Politi, F. Nudelman, S. Weiner, Biomineralization design strategies and mechanisms of mineral formation: operating at the edge of instability BT engineering of Crystalline Materials Properties, in: J.J. Novoa, D. Braga, L. Addadi (Eds.), NATO Sci. Peace Secur. Ser. B: Phys. Biophys. ,Eng. Crystall. Mater. Properties (2008) 1-15. Springer Netherlands.

[21] F. Marin, B. Pokroy, G. Luquet, P. Layrolle, K. De Groot, Protein mapping of calcium carbonate biominerals by immunogold, Biomaterials 28 (2007) 2368-2377, https://doi.org/10.1016/j.biomaterials.2007.01.029.

[22] Y. Dauphin, E. Zolotoyabko, A. Berner, E. Lakin, C. Rollion-Bard, J.P. Cuif, P. Fratzl, Breaking the long-standing morphological paradigm: individual prisms in the pearl oyster shell grow perpendicular to the c-axis of calcite, J. Struct. Biol. 205 (2019) 121-132, https://doi.org/10.1016/j.jsb.2019.01.004.

[23] F. Marin, G. Luquet, B. Marie, D. Medakovic, Molluscan shell proteins: primary structure, origin, and evolution, Curr. Top. Dev. Biol. 80 (2007) 209-276, https:// doi.org/10.1016/S0070-2153(07)80006-8.

[24] A.G. Checa, F.J. Esteban-Delgado, J. Ramírez-Rico, A.B. Rodríguez-Navarro, Crystallographic reorganization of the calcitic prismatic layer of oysters, J. Struct. Biol. 167 (2009) 261-270, https://doi.org/10.1016/j.jsb.2009.06.009.

[25] N. Sommerdijk, M. Cusack, Crystals competing for space, Nat. Mater. 13 (2014) 1078-1079, https://doi.org/10.1038/nmat4147.

[26] B. Bayerlein, P. Zaslansky, Y. Dauphin, A. Rack, P. Fratzl, I. Zlotnikov, Self-similar mesostructure evolution of the growing mollusc shell reminiscent of thermodynamically driven grain growth, Nat. Mater. 13 (2014) 1102-1107, https://doi.org/10.1038/nmat4110.

[27] A.G. Checa, E. Macías-Sánchez, E.M. Harper, J.H.E. Cartwright, Organic membranes determine the pattern of the columnar prismatic layer of mollusc shells, Proc. R. Soc. B Biol. Sci. 283 (2016), 20160032, https://doi.org/10.1098/ rspb.2016.0032.

[28] Y. Dauphin, A. Brunelle, K. Medjoubi, A. Somogyi, J.P. Cuif, The prismatic layer of Pinna: a showcase of methodological problems and preconceived hypotheses, Minerals 8 (2018) 365, https://doi.org/10.3390/min8090365.

[29] A.P. Jackson, J.F.V. Vincent, R.M. Turner, R.M. Alexander, The mechanical design of nacre, Proc. Roy. Soc. Lond. B. 234 (1988) 415-440, https://doi.org/10.1098/ rspb.1988.0056.

[30] F. Barthelat, H.D. Espinosa, An experimental investigation of deformation and fracture of nacre-mother of pearl, Exp. Mech. 47 (2007) 311-324, https://doi.org/ 10.1007/s11340-007-9040-1.

[31] R. Rabiei, A.K. Dastjerdi, F. Barthelat, in: B.C. Prorok, et al. (Eds.), Interfacial Fracture Toughness of Nacre BT - Mechanics of Biological Systems and Materials, ume 5, Springer, New York, 2013, pp. 31-38.

[32] J. Gim, N. Schnitzer, L.M. Otter, Y. Cui, S. Motreuil, F. Marin, S.E. Wolf, D.E. Jacob, A. Misra, R. Hovden, Nanoscale deformation mechanics reveal resilience in nacre of Pinna nobilis shell, Nat. Commun. 10 (2019) 1-8, https://doi.org/10.1038/ s41467-019-12743-z.

[33] J.D. Taylor, The mechanical properties of bivalve (Mollusca) shell structures, Paleontology 15 (1972) 73-87.

[34] X.W. Li, H.M. Ji, W. Yang, G.P. Zhang, D.L. Chen, Mechanical properties of crossedlamellar structures in biological shells: a review, J. Mech. Behav. Biomed. Mater. 74 (2017) 54-71, https://doi.org/10.1016/j.jmbbm.2017.05.022.

[35] M. Strag, Ł. Maj, M. Bieda, P. Petrzak, A. Jarzębska, J. Gluch, E. Topal, K. Kutukova, A. Clauser, W. Heyn, K. Berent, K. Nalepka, E. Zschech, A.G. Checa, K. Sztwiertnia, Anisotropy of mechanical properties of Pinctada margaritifera mollusk shell, Nanomaterials 10 (2020) 634, https://doi.org/10.3390/ nano10040634.

[36] M.E. Kunitake, L.M. Mangano, J.M. Peloquin, S.P. Baker, L.A. Estroff, Evaluation of strengthening mechanisms in calcite single crystals from mollusk shells, Acta Biomater. 9 (2013) 5353-5359, https://doi.org/10.1016/j.actbio.2012.09.030.

[37] C.F. Böhm, P. Feldner, B. Merle, S.E. Wolf, Conical nanoindentation allows azimuthally independent hardness determination in geological and biogenic minerals, Materials 12 (2019) 1630, https://doi.org/10.3390/ma12101630.

[38] A.G. Checa, Fabricational morphology of oblique ribs in bivalves, J. Morphol. 254 (2002) 195-209, https://doi.org/10.1002/jmor.10028.

[39] A.R.S. Gautam, J.M. Howe, A method to predict the orientation relationship, interface planes and morphology between a crystalline precipitate and matrix. Part I. approach, Philos. Mag. A 91 (2011) 3203-3227, https://doi.org/10.1080/ 14786435.2011.573817.

[40] J.H.P. De Bresser, C.J. Spiers, Strength characteristics of the $r, f$, and $c$ slip systems in calcite, Tectonophysics 272 (1997) 1-23, https://doi.org/10.1016/S0040-1951 (96)00273-9.

[41] M. Bruno, F.R. Massaro, L. Pastero, E. Costa, M. Rubbo, M. Prencipe, D. Aquilano, New estimates of the free energy of calcite/water interfaces for evaluating the equilibrium shape and nucleation mechanisms, Cryst. Growth Des. 13 (2013) 1170-1179, https://doi.org/10.1021/cg3015817.

[42] J.T. Hagan, Micromechanics of crack nucleation during indentations, J. Mater. Sci. 14 (1979) 2975-2980, https://doi.org/10.1007/BF00611482.

[43] F. Bachmann, R. Hielscher, H. Schaeben, Texture analysis with MTEX - free and open source software Toolbox, Solid State Phenom. 160 (2010) 63-68. 10.4028/ www.scientific.net/SSP.160.63.

[44] K. Nalepka, K. Sztwiertnia, P. Nalepka, Preferred orientation relationships at the $\mathrm{Cu} / \alpha-\mathrm{Al}_{2} \mathrm{O}_{3}$ interface: identification and theoretical explanation, Acta Mater. 104 (2016) 156-165, https://doi.org/10.1016/j.actamat.2015.11.014.

[45] B. Fultz, J.M. Howe, Transmission Electron Microscopy and Diffractometry of Materials, Springer-Verlag Berlin Heidelberg, 2013, https://doi.org/10.1007/9783-642-29761-8.

[46] E. Prince, International Tables for Crystallography Vol. C: Mathematical, Physical and Chemical Tables, Springer, Dordrecht, 2006, https://doi.org/10.1107/ 97809553602060000103.

[47] B.D. Beake, S.R. Goodes, J.F. Smith, Micro-Impact Testing: a new technique for investigating thin film toughness, adhesion, erosive wear resistance, and dynamic hardness, Surf. Eng. 17 (2001) 187-192, https://doi.org/10.1179/ 026708401101517755.

[48] J.R. Garcia-March, A. Márquez-Aliaga, J.G. Carter, The duplivincular ligament of recent Pinna Nobilis L., 1758: further evidence for pterineid ancestry of the Pinnoidea, J. Paleontol. 82 (2008) 621-627, https://doi.org/10.1666/06-096.1. 